\title{
Examples of conservative diffeomorphisms of the two-dimensional torus with coexistence of elliptic and stochastic behaviour
}

\author{
FELIKS PRZYTYCKI \\ Institute of Mathematics, Polish Academy of Sciences, ul. Sniadeckich 8, \\ 00-950 Warsaw, Poland
}

(Received 7 July 1982)

Dedicated to the memory of V. M. Alexeyev

Abstract. We find very simple examples of $C^{\infty}$-arcs of diffeomorphisms of the two-dimensional torus, preserving the Lebesgue measure and having the following properties: (1) the beginning of an arc is inside the set of Anosov diffeomorphisms; (2) after the bifurcation parameter every diffeomorphism has an elliptic fixed point with the first Birkhoff invariant non-zero (the KAM situation) and an invariant open area with almost everywhere non-zero Lyapunov characteristic exponents, moreover where the diffeomorphism has Bernoulli property; (3) the arc is real-analytic except on two circles (for each value of parameter) which are inside the Bernoulli property area.

Topologically after the bifurcation parameter we have hyperbolic toral automorphisms with 0 'blown up'.

\section{Introduction}

In this paper we find a simple one-parameter family of diffeomorphisms of the two-dimensional torus $T^{2}, H_{t}: T^{2} \rightarrow T^{2}$ for $t \in[-\varepsilon, \varepsilon]$, preserving the Lebesgue measure and satisfying the properties (1)-(5) listed below.

(1) For every $t>0, H_{t}$ is inside the set of Anosov diffeomorphisms $\mathrm{An}\left(T^{2}\right)$. For every $t \geq 0, H_{t}$ is topologically conjugate with the hyperbolic toral automorphism $A$ given by the matrix $\left(\begin{array}{ll}1 & 1 \\ 1 & 2\end{array}\right)$.

(2) The family $H_{t}$ at $t=0$ is transversal to the set Fr An $\left(T^{2}\right)-$ the boundary of An $\left(T^{2}\right)$. We mean by this that there exists a constant $C>0$ such that

$$
\operatorname{dist}_{C^{1}}\left(H_{t}, \operatorname{Fr} \operatorname{An}\left(T^{2}\right)\right) \geq C \cdot|t| \text {. }
$$

(3) For every $t<0$ there exists an elliptic island around $0 \in T^{2}$. This means that the differential $D H_{t}(0)$ is elliptic, the eigenvalues of $D H_{t}(0)$ are not roots of unity of low degree and in the Birkhoff normal form the frequency of oscillations depends on the amplitude. More exactly, the first Birkhoff invariant is non-zero. Then by Kolmogorov-Arnold-Moser theory most of the neighbourhood of 0 is filled with $H_{t}$-invariant closed curves. 
(4) For every $t<0$ there exists an open, non-empty $H_{t}$-invariant set $S_{t} \subset T^{2}$ on which $H_{t}$ behaves stochastically. More exactly the Lyapunov characteristic exponents for $\left.H_{t}\right|_{s_{t}}$ are almost everywhere non-zero and $H_{t}$ restricted to $S_{t}$ has the Bernoulli property.

(5) $H:[-\varepsilon, \varepsilon] \times T^{2} \rightarrow T^{2}$ is a $C^{\infty}$-function and is real-analytic except on the two families of circles $[-\varepsilon, \varepsilon] \times\{a, b\} \times S^{1}$.

We look for $H_{t}$ in the form of a toral-linked twist mapping, see [1] and [9], i.e.

where

$$
H_{t}=G_{\mathbf{g}_{t}} \circ F_{f_{t}},
$$

$$
\begin{gathered}
F_{f_{t}}(x, y)=\left(x+f_{t}(y), y\right), \quad G_{\mathrm{g}_{t}}(x, y)=\left(x, y+g_{t}(x)\right), \\
f_{t}, g_{t}: \mathbb{R} \rightarrow \mathbb{R}, \quad f_{t}(y+1)=f_{t}(y)+k, \\
g_{t}(x+1)=g_{t}(x)+l,
\end{gathered}
$$

for every $x, y \in \mathbb{R}$ and some integers $k$ and $l$.

We take $f_{t}=$ id so that

$$
H_{t}(x, y)=\left(x+y, y+g_{t}(x+y)\right)
$$

We take $g_{t}$ satisfying the following properties:

$\left\{\begin{array}{l}\text { For every } t \in[-\varepsilon, \varepsilon] g_{t} \text { is an odd function, } \\ g_{t}(0)=0, \quad g_{t}(1)=1, \quad d g_{0} / d x(0)=d^{2} g_{0} / d x^{2}(0)=0, \quad d^{3} g_{0} / d x^{3}(0)>0, \\ d^{2} g_{t} /\left.d x d t(x)\right|_{x=0, t=0}>0 \text { and } d g_{0} / d x(x)>0 \text { for every } x \notin Z .\end{array}\right.$

If $t>0$, the point $0 \in T^{2}$ is a saddle for $H_{t}$. When $t$ passes 0 in the negative direction two saddles $p_{t}$ and $q_{t}$ appear on the opposite sides of 0 on the $x$-axis while 0 itself becomes elliptic.

Checking the properties (1)-(3) is straightforward so we do not dwell on them. Let us only mention that $H_{0}$ corresponds to diffeomorphisms studied in [1] and [6] and that for $t<0,|t|$ sufficientiy small, the first Birkhoff invariant at $0 \in T^{2}$ is non-zero since $d^{3} g_{t} / d x^{3}(0) \neq 0$.

Thus, the main aim of the paper is to prove property (4) for a special family $g_{t}$.

In general the stable and unstable manifolds of $p_{t}$ and $q_{t}$ intersect transversally (see the phase portrait in figure 1) and in such a case we do not know how to estimate the Lyapunov exponents. Moreover, in view of a recent result by $R$. Mañé [7] there exists a $C^{1}$-generic subset

$$
\mathscr{A}_{L} \subset \operatorname{Diff}_{L}^{1}\left(T^{2}\right) \backslash \mathrm{An}_{L}\left(T^{2}\right)
$$

where Lyapunov exponents are zero almost everywhere. So our $H_{t}$ must be disjoint with $\mathscr{A}_{L}$. (The subscript $L$ means that we consider diffeomorphisms preserving the Lebesgue measure.) In connection with the Mañé result Katok has suggested studying the Lyapunov exponents for small perturbations of $H_{0}$.

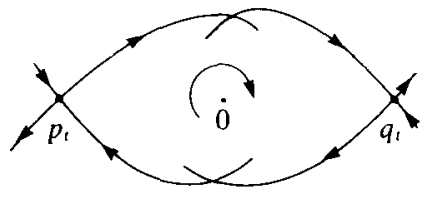

FIGURE 1

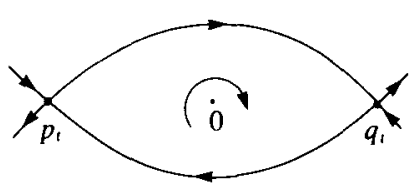

FIGURE 2 
In the case of our special $H_{t}$ the saddles $p_{t}$ and $q_{t}$ are joined by separatrices, see figure 2. Throughout the paper we use only this property of $H_{t}$, together with properties (1). In the theorem in $\S 2$, we consider a specific $H_{t}$ only to be concrete.

Denote the domain between the separatrices by $U_{t}$. An idea which explains property (4) is that $T^{2} \mid \mathrm{cl} U_{t}$ is $H_{t}$-invariant so the behaviour along the trajectory of every point from $T^{2} \mid \mathrm{cl} U_{t}$ is hyperbolic as the trajectory keeps far away from the elliptic island around $0 \in T^{2}$.

In fact we 'blow up' the saddle of the Anosov diffeomorphism into the disk $\operatorname{cl} U_{t}$. We use the Hamiltonian function $y^{2}-x^{2}\left(x^{2}+2 t\right)$. In a neighbourhood of $\operatorname{cl} U_{t}$ the saddle-like dynamics are preserved.

Section 2 is devoted to the construction of $H_{t}$. In $\S \S 3-5$ we prove property (4) using the technique of invariant cones. In $\$ 6$ we prove that for each $t<0,\left.H_{t}\right|_{T^{2}|c| U_{t}}$ is an almost Anosov diffeomorphism. Namely, it has continuous, uniquely integrable stable and unstable sub-bundles; it has almost everywhere non-zero Lyapunov exponents for every $H_{t}$ invariant probability measure on $T^{2} \mid \mathrm{cl} U_{t}$ and it is topologically conjugate to the Anosov diffeomorphism $\left.A\right|_{T^{2} \backslash\{0\}}$. However proposition $3, \S 6$ proves that our 'blowing up' is in no sense $C^{1}$. Our study in $\$ 6$ corresponds to the Katok study for the $H_{0}$-type example [6] and to the Gerber and Katok study of smoothed pseudo-Anosov diffeomorphisms [4].

One reason why it is easy to construct our examples of coexistence is that we perturb the twist $F_{\text {id }}$ with $G_{g_{t}}$ where $g_{t}$ is not periodic, i.e. the average twisting

$$
\int_{0}^{1} \frac{d g_{t}}{d x}(x) d x \neq 0
$$

The classical problem is to consider $g_{t}$ to be periodic. Nevertheless the facts of local character i.e. the dynamics in the neighbourhood of $\mathrm{cl} U_{t}$, like lemmas 2,3 , 5 , concern the classical situation. See $\$ 7$ for further comments.

\section{Construction of the example}

THEOREM. Let $H_{t}$ be the one-parameter family of diffeomorphisms

with $\mathrm{g}_{\mathrm{t}}$ defined as follows:

$$
H_{t}=H_{\mathrm{id}, \mathrm{g}_{\mathrm{t}}}: T^{2} \rightarrow T^{2} \text { for } t \in[-\varepsilon, \varepsilon] \text {, }
$$

$$
g_{t}(x)=2(\sqrt{1-t+2 x}-\sqrt{1-t-2 x}-2 x), \text { for }|x| \leq \frac{1}{4} ;
$$

$g_{t}$ is extended to $\left[-\frac{1}{4}, \frac{1}{4}\right]+Z$ by

$$
g_{t}(x+n)=g_{t}(x)+n \quad \text { for } x \in\left[-\frac{1}{4}, \frac{1}{4}\right], n \in Z
$$

and extended to $] \frac{1}{4}, \frac{3}{4}[+Z$ in anyway so that

$$
\begin{aligned}
& \text { inf }\left\{d g_{t} / d x(x): x \in\right]^{\frac{1}{4}}, \frac{3}{4}[\}=d g_{t} / d x\left(\frac{1}{4}\right) ; \\
& g_{t}-\mathrm{id} \text { is periodic with period } 1 ; \\
& g:[-\varepsilon, \varepsilon] \times \mathbb{R} \rightarrow \mathbb{R} \text { is } C^{\infty} \text { and } \\
& \left.\quad \mathrm{g}\right|_{[-\varepsilon, \varepsilon] \times\left(\mathbb{R} \backslash\left(\frac{1}{2} \cdot z+\frac{1}{4}\right)\right)} \text { is real-analytic. }
\end{aligned}
$$

Then the family $H_{t}$ satisfies properties (1)-(5) from the introduction.

For example, for $x \in] \frac{1}{4}, \frac{3}{4}[$ set

$$
g_{t}(x)=g_{t}\left(\frac{1}{4}\right)+\left(x-\frac{1}{4}\right) \cdot \frac{d g_{t}}{d x}\left(\frac{1}{4}\right)+\left(\int_{\frac{1}{4}}^{x} \varphi(s) d s\right)\left(1-2 g_{t}\left(\frac{1}{4}\right)-\frac{1}{2} \frac{d g_{t}}{d x}\left(\frac{1}{4}\right) / \int_{\frac{1}{4}}^{\frac{3}{4}} \varphi(s) d s\right),
$$


where

$$
\varphi(x)=\exp \left(\sin 2 \pi\left(x+\frac{1}{4}\right)\right)^{-1} .
$$

Let us consider the following one-parameter family of Hamiltonian functions defined in the neighbourhood of $t=x=y=0$ :

$$
h_{t}(x, y)=y^{2}-x^{2}\left(x^{2}+2 t\right) \text {. }
$$

For $t>0$ the Hamiltonian vector field $V_{t}$ corresponding to $h_{t}$ has a saddle at $0 \in T^{2}$. For $t<0$ this saddle changes into an elliptic fixed point and $V_{t}$ acquires two saddles

$$
p_{t}=(-\sqrt{|t|}, 0), \quad q_{t}=(\sqrt{|t|}, 0)
$$

joined by two separatrices, see figure 2 in $\S 1$. We look for $g_{t}$ such that

$$
F_{2 \mathrm{id}} \circ H_{\mathrm{id}, \mathbf{g}_{t}} \circ F_{\mathbf{2}}^{-1}
$$

has the same saddles and separatrices.

The union of stable and unstable manifolds for the saddles $p_{t}$ and $q_{t}$ in the neighbourhood of $0 \in T^{2}$ coincides with the set of zeros of the function:

$$
\begin{aligned}
h_{t}(x, y)-h_{t}\left(p_{t}\right) & =y^{2}-x^{2}\left(x^{2}+2 t\right)-t^{2} \\
& =y^{2}-\left(x^{2}+t\right)^{2} \\
& =-\left(x^{2}+t+y\right)\left(x^{2}+t-y\right) .
\end{aligned}
$$

Consider the set of zeros of $W_{t}(x, y)=x^{2}+t+y$ and then the zeros of $W_{t}\left(x \pm \frac{1}{2} y, y\right)$ (broken lines in figure 3). Write these sets as graphs of the functions

$$
y_{t}^{ \pm}(x)=2(-1 \mp x+\sqrt{1 \pm 2 x-t}) \text {. }
$$

Define $g_{t}=y_{t}^{+}-y_{t}^{-}$. We obtain the formula from the statement of the theorem.

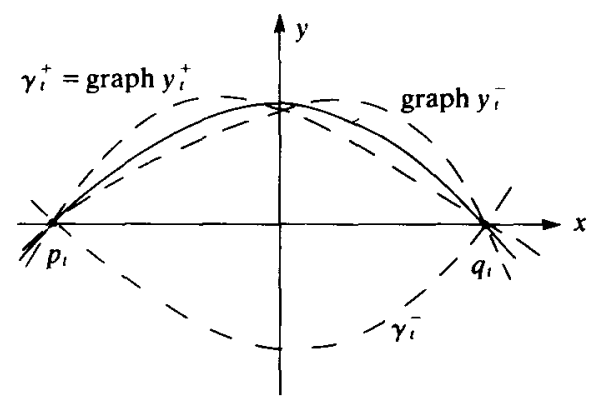

FIGURE 3

From the construction:

$$
F_{\text {id }}\left(\text { graph } y_{t}^{+}\right)=\operatorname{graph} y_{t}^{-} \text {and } G_{g_{t}}\left(\operatorname{graph} y_{t}^{-}\right)=\operatorname{graph} y_{t}^{+} .
$$

So our goal has been reached: $H_{\mathrm{id}_{\mathrm{gt}}}$ has the separatrices

$$
\gamma_{t}^{+}=\text {graph } y_{t}^{+} \text {and } \gamma_{t}^{-}=-\gamma_{t}^{+} \text {. }
$$

They are $F_{\frac{1}{2 i d}}^{-1}$ images of the separatrices of $V_{t}$.

The vectors $(1 \mp \sqrt{|t|}, \pm 2 \sqrt{|t|})$ are eigenvectors of $p_{t}$ and $q_{t}$. The corresponding eigenvalues are

$$
(1+\sqrt{|t|}) /(1-\sqrt{|t|}) \text { and }(1-\sqrt{|t|}) /(1+\sqrt{|t|})
$$


These numbers will appear throughout the paper. Sometimes we shall use the notation $(v)_{x},(v)_{y},(z)_{x},(z)_{y}$ to denote the $x$ - or $y$-coordinate of a vector $v$ or of a point $z$.

\section{Existence of invariant families of cones}

We shall describe here families of unstable and stable cones in the region $T^{2} \mid \mathrm{cl} U_{t}$, where $U_{t}$ is the region between the separatrices $\gamma_{t}^{ \pm}$.

Denote for every $a<b,|a-b|<1$, the strip $] a, b\left[\times S^{1}\right.$ by $P(a, b)$.

Denote by $\mathscr{T}_{t}(\delta)$ the region ('triangle') bounded by the components of the stable and unstable manifolds of $p_{t}$ in $\operatorname{cl} P(-\sqrt{|t|}-\delta,-\sqrt{|t|})$ containing $p_{t}$ and the line

$$
\{x=-\sqrt{|t|}-\delta\} \quad \text { for any small } \delta>0
$$

and denote $\mathscr{T}_{\mathfrak{t}}^{\prime}(\delta)=-\mathscr{T}_{\imath}(\delta)$ (see figure 4).

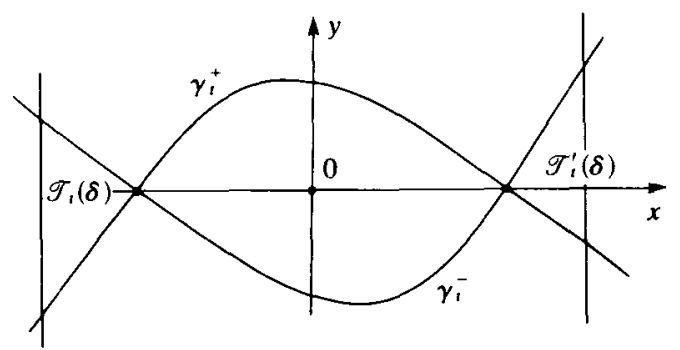

Figure 4

Let us start now with

LEMMA 1. There exists a constant $C_{1}>0\left(C_{1} \ll 1\right)$ such that for every $\delta: 0<\delta \leq C_{1}$ and $t \in\left[-\varepsilon, 0\left[\right.\right.$, if $z, H_{t} z \in P(-\delta, \delta) \backslash\left(\mathrm{cl} U_{t} \cup \operatorname{Fr} \mathscr{T}_{t}(\delta) \cup \operatorname{Fr} \mathscr{T}_{t}^{\prime}(\delta)\right)$ then there exists integers $N_{1}>0, N_{2}>1$ such that

$$
H_{t}^{-N_{1}}(z), \quad H_{t}^{N_{2}}(z) \in \operatorname{cl} P(\delta, 1-\delta) \quad \text { and } \quad H_{t}^{n}(z) \in P(-\delta, \delta)
$$

for every $n:-N_{1}<n<N_{2}$, and one of the following possibilities occurs :

(1) $z_{n} \in \mathscr{T}_{t}(\delta-\sqrt{|t|})$ for every $n:-N_{1}<n<N_{2}$ where by definition

$$
z_{n}=\left(x_{n}, y_{n}\right)=H_{t}^{n}(z) ;
$$

(2) $z_{n} \in \mathscr{T}_{t}^{\prime}(\delta-\sqrt{|t|})$ for every $n:-N_{1}<n<N_{2}$;

(3) $0<y_{n}<2 \delta+\sup \left\{g_{t}(x): x \in[-\delta, \delta]\right\}$ for $-N_{1}<n<N_{2}$ and the sequence $\left(x_{n}\right), n=-N_{1}, \ldots, N_{2}$ is increasing;

(4) $0>y_{n}>-2 \delta+\inf \left\{g_{t}(x): x \in[-\delta, \delta]\right\}$ for $-N_{1}<n<N_{2}$ and the sequence $\left(x_{n}\right), n=-N_{1}, \ldots, N_{2}$ is decreasing.

The proof is straightforward so it is omitted.

For $t<0$ denote by $a_{t}$ the smallest positive number such that

$$
d g_{t} / d x\left(a_{t}\right)=4 \sqrt{|t|} /(1-\sqrt{|t|}) .
$$

Remark 1 . There is no need to compute $a_{t}$ exactly. Observe only that $a_{t}$ exists and it is of order $\sqrt[4]{|t|}$ since

$$
g_{t}(x)=2 Q(t, x)\left(x^{3}+t x\right) \quad \text { where } Q(0,0)=1 .
$$


This follows easily from the definition of $g_{t}(x)$.

For every $x$ such that $|x| \leq \sqrt{|t|}$ denote by $\mathscr{C}(x)$ the cone:

$$
\mathscr{C}(x)=\left\{(\xi, \eta) \in \mathbb{R}^{2}: d y_{t}^{+} / d x(x) \leq \eta / \xi\right\} .
$$

For every $z \in T^{2}$ we shall identify the tangent space $T_{z}\left(T^{2}\right)$ with $\mathbb{R}^{2}$. Define now $\mathscr{D}(z) \subset T_{z}\left(T^{2}\right)$ for every $z=(x, y) \in T^{2} \mid \mathrm{cl} U_{t}$ as follows:

(i) $\mathscr{D}(z)=\mathscr{C}(-\sqrt{|t|})$ if $z \in \operatorname{cl} P\left(a_{t}, 1-a_{t}\right)$;

(ii) $\mathscr{D}(z)=\mathscr{C}(-\sqrt{|t|}) \quad$ if $z \in \mathscr{P}_{t}=P\left(-a_{t},-\sqrt{|t|}\right) \cup P\left(\sqrt{|t|}, a_{t}\right)$;

and the backward trajectory $H_{t}^{-n}(z), n=1,2, \ldots$, either hits cl $P\left(a_{t}, 1-a_{t}\right)$ earlier than $\mathrm{cl} P(-\sqrt{|t|}, \sqrt{|t|})$ or never hits $\mathrm{cl} P(-\sqrt{|t|}, \sqrt{|t|})$;

(iii) $\mathscr{D}(z)=\mathscr{C}(\sqrt{|t|})$ if as in case (ii) $z \in \mathscr{P}_{t}$ but hits the set $\operatorname{cl} P(-\sqrt{|t|}, \sqrt{|t|})$ earlier than $P\left(a_{t}, 1-a_{t}\right)$;

(iv) $\mathscr{D}(z)=\mathscr{C}(\sqrt{|t|})$ if $z \in \operatorname{cl} P(-\sqrt{|t|}, \sqrt{|t|}), H_{t}(z) \notin P(-\sqrt{|t|}, \sqrt{|t|})$;

(v) $\mathscr{D}(z)=\mathscr{C}(x)$ if $z, H_{t}(z) \in P(-\sqrt{|t|}, \sqrt{|t|})$ and $y>0 \quad(y>0$ makes sense since, by lemma $1,|y|$ is small);

(vi) $\mathscr{D}(z)=\mathscr{C}(-x)$ if in (v) we replace $y>0$ by $y<0$.

Now we are going to prove the invariance of this cone bundle. If $z$ and $H_{t}(z)=\left(x_{1}, y_{1}\right)$ are as in cases (i) or (ii) then

$$
\frac{d g_{t}}{d x}\left(x_{1}\right) \geq \frac{d g_{t}}{d x}(-\sqrt{|t|})
$$

so

$$
\begin{aligned}
\left(D H_{t}\right)_{z}(\mathscr{D}(z)) & =\left(D H_{t}\right)_{z}(\mathscr{C}(-\sqrt{|t|})) \subset\left(D H_{t}\right)_{p_{t}}(\mathscr{C}(-\sqrt{|t|})) \\
& =\mathscr{C}(-\sqrt{|t|})=\mathscr{D}\left(H_{t}(z)\right) .
\end{aligned}
$$

If $z=(x, y)$ as in (i) or (ii) and $H_{t}(z)=\left(x_{1}, y_{1}\right)$ as in (v) (or similarly (vi)), then we use the concavity of the function $y_{t}^{+}$. Let $z_{1}^{\prime}=\left(x_{1}, y_{1}^{\prime}\right)$ be the point on the same vertical as $\left(x_{1}, y_{1}\right)$, lying in the $\gamma_{t}^{+}$(see figure 5). Let $H_{t}^{-1}\left(z_{1}^{\prime}\right)=\bar{z}=(\bar{x}, \bar{y})$. Then

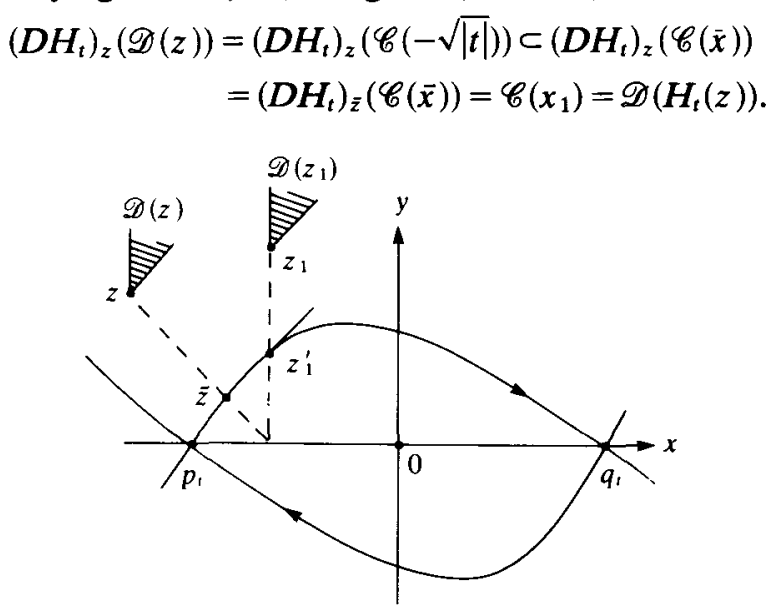

Figure 5

If $z$ and $H_{t}(z)$ are both as in (v) (or (vi)) the argument is similar.

It is also similar for $z$ given by (v) or (vi) and $H_{t}(z)$ given by (iv). 
If $z$ is as in (iv) or (iii) and $H_{t}(z)$ as in (iii), then analogously to the first-considered case:

$$
\begin{aligned}
\left(D H_{t}\right)_{z}(\mathscr{D}(z)) & =\left(D H_{t}\right)_{z}(\mathscr{C}(\sqrt{|t|})) \subset\left(D H_{t}\right)_{q_{t}}(\mathscr{C}(\sqrt{|t|})) \\
& =\mathscr{C}(\sqrt{|t|})=\mathscr{D}\left(H_{t}(z)\right) .
\end{aligned}
$$

Finally if $z$ is as in (iii) or (iv) and $H_{t}(z)=\left(x_{1}, y_{1}\right)$ as in (i), then by (2) we have

$$
d g_{t} / d x\left(x_{1}\right) \geq 4 \sqrt{|t|} /(1-\sqrt{|t|})
$$

hence

$$
\left(D H_{t}\right)_{z}(\mathscr{D}(z))=\left(D H_{t}\right)_{z}(\mathscr{C}(\sqrt{|t|})) \subset \mathscr{C}(-\sqrt{|t|})=D\left(H_{t}(z)\right)
$$

Note that due to lemma 1 it cannot happen that $z$ is as in case (iii) and in the same time $H_{t}(z)$ as in cases (iv), (v) or (vi).

So the invariance of this cone bundle has been proved. We have the cone bundle $\mathscr{D}$ over $T^{2} \mid \mathrm{cl} U_{t}$ and

$$
D H_{t}(\mathscr{D}) \subset \mathscr{D} \text {. }
$$

The analogous stable cone bundle $\mathscr{D}^{s}$ i.e. such that $D H_{t}^{-1}\left(\mathscr{D}^{s}\right) \subset \mathscr{D}^{s}$ can be defined by

$$
\mathscr{D}^{s}=D\left(S_{\mathrm{y}} \circ F_{\text {id }}\right)(\mathscr{D})
$$

where $S_{\mathrm{y}}$ is the symmetry with respect to the $y$-axis.

Remark 2. At this stage we can immediately deduce the existence of a set of positive Lebesgue measure with non-zero Lyapunov characteristic exponents as follows.

The set of line sub-bundles of $\mathrm{cl}(\mathscr{D})$ over $T^{2} \mid \mathrm{cl} U_{t}$ is a partially ordered set, with angle order over every point. Take the bundle $L(\partial / \partial y)$ spanned by the vector field $\partial / \partial y$. For every $z \in T^{2} \mid \mathrm{cl} U_{t}$,

$$
L(\partial / \partial y)(z) \in \operatorname{cl} \mathscr{D}(z)
$$

and the sequence $D H_{t}^{n}(L(\partial / \partial y))$ is monotonous with respect to the considered partial order. Hence the pointwise limit, a measurable line bundle, is a fixed point for $D H_{t}$ (see [2, theorem 3.8.1] for the details). Denote this bundle by $E_{t}$. Now use the Birkhoff ergodic theorem for the function $\left\|\left.D H_{t}\right|_{E_{t}}\right\|$.

Let $\lambda: T^{2} \backslash \mathrm{cl} U_{t} \rightarrow \mathbb{R}$ be the Lyapunov characteristic exponent for the vectors from $E_{\text {t. } \text { Then }}$

$$
\int_{T^{2} \backslash \mathrm{cl} U_{\mathrm{t}}} \lambda(z) d z=\int_{T^{2} \backslash \mathrm{cl} U_{\mathrm{t}}} \log \left\|\left.D H_{t}\right|_{E_{\mathrm{t}}}(z)\right\| d z
$$

which is clearly positive for $|t|$ sufficiently small. So $\lambda(z)$ is positive on a set of positive Lebesgue measure. The second Lyapunov exponent, which is equal to $-\lambda(z)$, is negative on the same set.

4. Lyapunov characteristic exponents are non-zero almost everywhere on $T^{2} \backslash \mathrm{cl} U_{t}$

LEMMA 2. Let

$$
\begin{gathered}
z=(x, y) \in \operatorname{cl} P(-\sqrt{|t|}, 0) \mid \operatorname{cl} U_{t} \\
H_{t}(z)=\left(x_{1}, y_{1}\right) \in \operatorname{cl} P(0, \sqrt{|t|}) \\
y, y_{1}>0 \quad \text { and } \quad|x|<\left|x_{1}\right| .
\end{gathered}
$$


As in lemma 1 let us put

$$
H_{t}^{n}(z)=z_{n}=\left(x_{n}, y_{n}\right) \quad \text { for } n \in Z .
$$

Then for every $n \geq 1$

$$
\left|x_{-n+1}\right| \leq\left|x_{n}\right| \leq\left|x_{-n}\right| \text {. }
$$

Proof. Observe that the backward (i.e. forward under $\left.H_{t}^{-1}\right) H_{t}$-trajectory of the point $z_{0}$ is the reflection in the $y$-axis of the forward trajectory under $F_{\mathrm{id}} \circ G_{\mathrm{gt}_{\mathrm{t}}}$ of the point $\left(-x_{0}, y_{0}\right)$. So the latter trajectory is the sequence of points $\left(-x_{-n}, y_{-n}\right)$.

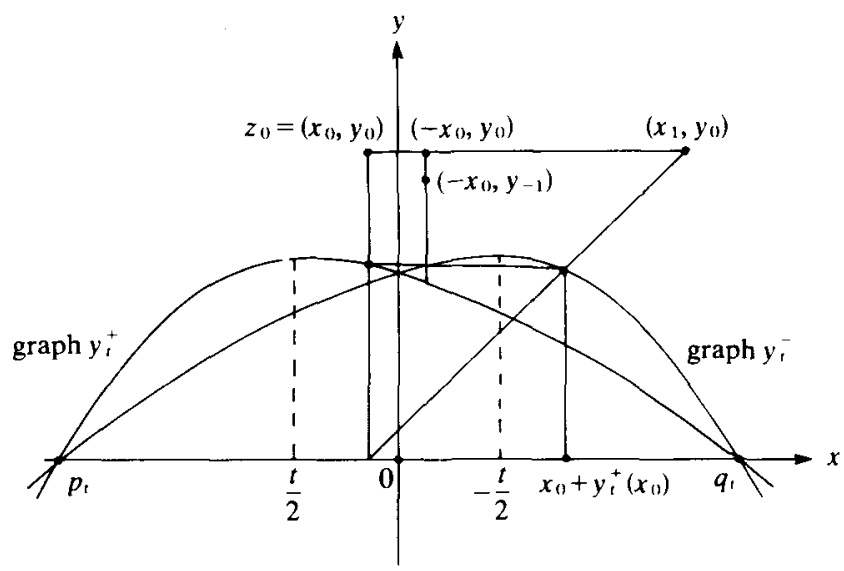

FIGURE 6

Assume that $x_{0} \geq t / 2$. At $t / 2$ the function $y_{t}^{+}$reaches its maximum (see figure 6). We have

$$
\begin{aligned}
y_{0}-y_{t}^{-}\left(-x_{0}\right) & =y_{0}-y_{t}^{+}\left(x_{0}\right) \\
& =y_{0}-y_{t}^{-}\left(x_{0}+y_{t}^{+}\left(x_{0}\right)\right)<y_{0}-y_{t}^{-}\left(x_{1}\right),
\end{aligned}
$$

since $x_{1}>x_{0}+y_{t}^{+}\left(x_{0}\right)$ and the function $y_{t}^{-}$is decreasing to the right from $x_{0}+y_{t}^{+}\left(x_{0}\right)$.

If $x_{0}<t / 2$ we have again

$$
y_{0}-y_{t}^{-}\left(-x_{0}\right) \leq y_{0}-y_{t}^{-}\left(x_{1}\right)
$$

since by our assumptions $-t / 2<-x_{0} \leq x_{1}$.

In the case $-x_{0}=x_{1}$ the lemma is trivially true so we can assume that $-x_{0}<x_{1}$. Joint the points $\left(-x_{0}, y_{0}\right)$ and $\left(x_{1}, y_{0}\right)$ by a curve $\alpha:[0,1] \rightarrow \mathbb{R}^{2}$ which is the interval in the coordinates $\left(x, y-y_{t}^{-}(x)\right)$. Due to (4), for every $s_{0} \in[0,1]$,

$$
D \alpha\left((\partial / \partial s)\left(s_{0}\right)\right) \in\left(D F_{\text {id }}(\mathscr{D})\right)\left(\alpha\left(s_{0}\right)\right),
$$

so that for every $n \geq 0$

$$
D\left(H_{t}^{n} \circ G_{\mathrm{g}_{t}} \circ \alpha\right)\left((\partial / \partial s)\left(s_{0}\right)\right) \in \mathscr{D}\left(H_{t}^{n} \circ G_{\mathbf{g}_{t}} \circ \alpha\left(s_{0}\right)\right)
$$

and the $x$-coordinate

$$
\left(D\left(H_{t}^{n} \circ G_{\mathbf{g}_{r}} \circ \alpha\right)\left((\partial / \partial s)\left(s_{0}\right)\right)\right)_{x}>0 .
$$

Hence $x_{n}>-x_{-n+1}$ for $n>0$. This proves the left hand side inequality in (3). 
To prove the right hand side inequality we observe that

$$
y_{0}-y_{t}^{+}\left(x_{0}\right)=y_{0}-y_{t}^{-}\left(-x_{0}\right)=y_{-1}-y_{t}^{+}\left(-x_{0}\right) \text {. }
$$

We join the points $\left(x_{0}, y_{0}\right)$ and $\left(-x_{0}, y_{-1}\right)$ by the interval in the coordinates $\left(x, y-y_{t}^{+}(x)\right)$ (unless $x_{0}=0$, which is the trivial case) and then proceed as before.

LEMMA 3. For every $\delta>0$ such that $\sqrt{|t|}+\delta \leq C_{1}$, where $C_{1}$ is the constant from lemma 1, if

$$
z \in \operatorname{cl} P(\sqrt{|t|}+\delta, 1-\sqrt{|t|}-\delta)
$$

and $n(z)>0$ is the first integer such that

$$
H_{t}^{n(z)}(z) \in \operatorname{cl} P(\sqrt{|t|}+\delta, 1-\sqrt{|t|}-\delta),
$$

if $v \in \mathscr{D}(z)$, then

$$
\left(D H_{t}^{n(z)}(v)\right)_{x} \geq(1-6 \sqrt{|t|}) \cdot\left(D H_{t}(v)\right)_{x} .
$$

Proof. We can assume that $n(z) \geq 4$. Put

$$
H_{t}^{n}(z)=z_{n}=\left(x_{n}, y_{n}\right),
$$

assume for example that $y_{n}>0(n=0,1, \ldots n(z))$, i.e. the sequence $\left(x_{n}\right)$ is increasing (see lemma 1). It is possible because the case $y_{n}<0$ is similar, and if $n(z)<4$ or

$$
y_{n} \in \mathscr{T}_{t} \cup \mathscr{T}_{t}^{\prime}
$$

the lemma is true for obvious reasons.

Put $D H_{t}^{n}(v)=v_{n}=\left(\xi_{n}, \eta_{n}\right)$ and $l_{n}=\xi_{n+1} / \xi_{n}$. Using the fact that $v_{n} \in \mathscr{D}$ and the description of $\mathscr{D}$ from $\S 3$ we obtain the following estimates.

If $z_{n} \in P(-\sqrt{|t|}-\delta,-\sqrt{|t|}), \quad l_{n}>(1+\sqrt{|t|}) /(1-\sqrt{|t|})$

if $z_{n} \in \operatorname{cl} P(-\sqrt{|t|}, \sqrt{|t|}), \quad l_{n} \geq 1+d y_{t}^{+} / d x\left(x_{n}\right)$;

if $z_{n} \in \operatorname{cl} P(\sqrt{|t|}, \sqrt{|t|}+\delta), \quad l_{n} \geq(1-\sqrt{|t|}) /(1+\sqrt{|t|})$.

Now look what happens to $v_{n}$ under $D H_{t}^{-1}$. Equivalently, consider $D G_{\mathbf{g}_{\mathrm{t}}}^{-1}\left(v_{n+1}\right)$ under $D F_{\text {id }}^{-1}$. For

$$
z_{n+1} \in \operatorname{cl} P(-\sqrt{|t|}, \sqrt{|t|})
$$

we obtain

$$
l_{n}^{-1} \leq 1-d y_{\imath}^{-} / d x\left(x_{n+1}\right)
$$

hence

$$
l_{n} \geq\left(1+d y_{t}^{+} / d x\left(-x_{n+1}\right)\right)^{-1} .
$$

Let $n_{2}=n_{2}(z), n_{1}=n_{1}(z)$ and $n_{3}=n_{3}(z)$ be respectively the smallest non-negative integers such that $x_{n} \geq-\sqrt{|t|}, x_{n}>0$ and $x_{n}>\sqrt{|t|}$. Now make two additional assumptions:

$$
\begin{gathered}
n_{3}(z)-n_{2}(z) \geq 3 ; \\
\left|x_{n_{1}-1}\right| \leq\left|x_{n_{1}}\right| .
\end{gathered}
$$

Due to (5) and (6) the point $z_{n_{1}-1}$ satisfies the assumptions of lemma 2 about $z$. Hence, for every $k:-1 \leq k \leq n_{3}-3-n_{1}$

$$
l_{n_{1}+k} \cdot l_{n_{1}-k-3} \geq\left(1+\frac{d y_{t}^{+}}{d x}\left(-x_{n_{1}+k+1}\right)\right)^{-1}\left(1+\frac{d y_{t}^{+}}{d x}\left(x_{n_{1}-k-3}\right)\right) \geq 1 \text {. }
$$


We used here the fact that by lemma 2

$$
\left|x_{n_{1}+k+1}\right| \leq\left|x_{n_{1}-k-3}\right|
$$

and that the function $d y_{i}^{+} / d x$ is defined and decreasing between $x_{n_{1}-k-3}$ and $-x_{n_{1}+k+1}$. It is defined because by the left hand inequality in (3)

$$
\left|x_{n_{1}-k-3}\right| \leq\left|x_{n_{1}+k+2}\right| \leq\left|x_{n_{3}-1}\right| \text { for } k \leq n_{3}-3-n_{1} .
$$

We know, also by lemma 2 , that $\left|n_{2}-1-\left(n(z)-n_{3}\right)\right| \leq 1$. So

$$
\begin{aligned}
\xi_{n(z)} / \xi_{1} & =\prod_{i=1}^{n(z)-1} l_{i}=\prod_{i=n_{2}(+1)}^{n_{3}-3} l_{i} \cdot\left(l_{n_{2}}\right) \cdot l_{n_{3}-2} \cdot l_{n_{3}-1} \cdot \prod_{i=1}^{n_{2}-1} l_{i} \cdot \prod_{i=n_{3}}^{n(z)-1} l_{i} \\
& \geq\left(\frac{1-\sqrt{|t|}}{1+\sqrt{|t|}}\right)^{3}>1-6 \sqrt{|t|} .
\end{aligned}
$$

(We put the terms +1 and $l_{n_{2}}$ into parentheses because they appear only in the case $n_{3}-n_{1}=n_{1}-n_{2}-1$ and do not appear if $n_{3}-n_{1}=n_{1}-n_{2}$.)

In the case when (6) is not satisfied i.e. if $\left|x_{n_{1}-1}\right|>\left|x_{n_{1}}\right|$ we consider the reflection in the $y$-axis of the $F_{\text {id }} \circ G_{g_{t}}$-trajectory $\left(-x_{-n}, y_{-n}\right)$ or the $H_{t}$-trajectory $z_{n}=\left(-x_{-n}, y_{-n-1}\right)$. We can use lemma 2 for $\left(z_{n}\right)$, so we obtain for every $k \geq 0$

$$
\left|x_{n_{1}+k}\right| \leq\left|x_{n_{1}-k-1}\right| \leq\left|x_{n_{1}+k+1}\right| \text {. }
$$

This also gives $\xi_{n}(z) / \xi_{1}>1-6 \sqrt{|t|}$. The only difference in computation is that the term $l_{n_{1}-1}$ has no pair, see (7). But clearly $\left|x_{n_{1}-1}\right|>|t| / 2$, hence $l_{n_{1}-1} \geq 1$.

We eliminate assumption (5) in the following way.

$$
\eta_{n_{2}-1} / \xi_{n_{2}-1} \geq 2 \sqrt{|t|} /(1-\sqrt{|t|}),
$$

i.e. it is of the order of at least $\sqrt{|t|}$. Inf $d g_{t} / d x \geq 3 t$ for $t<0$ and $|t|$ sufficiently small. This follows easily from the representation

$$
g_{t}=Q(t, x) \cdot 2\left(x^{3}+t x\right), \quad \text { with } Q(0,0)=1,
$$

see remark 1 in $\$ 3$. Thus

hence if $\eta_{n} / \xi_{n} \geq K \sqrt{|t|}$, then

$$
\eta_{n+1} / \xi_{n+1}=\left(\eta_{n} /\left(\xi_{n}+\eta_{n}\right)\right)+d g_{t} / d x\left(x_{n+1}\right),
$$

$$
\eta_{n+1} / \xi_{n+1} \geq \min \left(\frac{1}{2} \cdot K, \frac{1}{2}\right) \cdot \sqrt{|t|}-3|t| .
$$

If we fix any integer $N>0$ and proceed by induction starting with $k=n_{2}-1$ we can prove that for every $k$ :

$$
n_{2}-1 \leq k \leq n_{2}+N
$$

$\eta_{k} / \xi_{k}$ is of the order of $\sqrt{|t|}$ for $|t|$ sufficiently small (depending on $N$ ), hence $\eta_{k} / \xi_{k}>0$. In particular, we can take

$$
N=n_{3}-n_{2}<3 \text {. }
$$

Then for $n: n_{2}-1 \leq n \leq n_{3}-1$,

$$
l_{n}=\left(\xi_{n}+\eta_{n}\right) / \xi_{n} \geq 1 .
$$

For all other $n$ we have trivially $l_{n} \geq 1$. This proves the lemma. 
Now we can estimate the Lyapunov exponents. Take the constant $C_{1}$ from lemma 1. Let $\alpha\left(C_{1}\right)>0$ be a constant such that

$$
\alpha\left(C_{1}\right)<\inf \left\{d g_{0} / d x(x): x \in\left[C_{1}, 1-C_{1}\right]\right\} .
$$

Then the similar inequality

$$
\alpha\left(C_{1}\right)<\inf \left\{d g_{\mathrm{t}} / d x(x): x \in\left[C_{1}, 1-C_{1}\right]\right\}
$$

holds for every $t$ with $|t|$ sufficiently small.

Put $Q=\operatorname{cl} P\left(C_{1}, 1-C_{1}\right)$. If $|t|$ is sufficiently small we can replace the cones $\mathscr{D}$ over $Q$ by smaller cones

$$
\mathscr{D}_{Q}=\left\{(\xi, \eta): \eta / \xi \geq-2 \sqrt{|t|} /(1-\sqrt{|t|})+\alpha\left(C_{1}\right)\right\}
$$

and leave the old cones over the complement of $Q$. Then clearly the new system of cones $\mathscr{D}^{\prime}$ is also $D H_{t}$-invariant.

For $v \in \mathscr{D}^{\prime}(z), z \in Q$ and $n(z)>0$ the first time when $H_{t}^{n(z)}(z) \in Q$, we have by lemma 3

$$
\begin{aligned}
\left(D H_{t}^{n(z)}(v)\right)_{x} /(v)_{x} & =\left(\left(D H_{t}^{n(z)}(v)\right)_{x} /\left(D H_{t}(v)\right)_{x}\right) \cdot\left(\left(D H_{t}(v)\right)_{x} /(v)_{x}\right) \\
& \geq(1-6 \sqrt{t}) \cdot\left(1-2 \sqrt{t} /(1-\sqrt{|t|})+\alpha\left(C_{1}\right)\right)=\lambda_{t}>1
\end{aligned}
$$

for $|t|$ sufficiently small.

This proves that for the first return mapping $\left(H_{t}\right)_{Q}$, for almost every $z \in Q$ one of the Lyapunov exponents is not less than $\log \lambda_{t}$, i.e. positive and the second one is negative.

It can be easily proved by use of the Birkhoff ergodic theorem that almost every point from $Q$ returns to $Q$ with positive frequency, see [1] for example. Hence also for almost every point from the set $\bigcup_{n=-\infty}^{+\infty} H_{\mathrm{t}}^{n}(Q)$ the Lyapunov characteristic exponents are non-zero. But the latter set by lemma 1 is equal to $T^{2} \mid \mathrm{cl} U_{t}$. This finishes the proof that Lyapunov exponents for $\left.H_{t}\right|_{T^{2} \mid c l} U_{t}$ are non-zero.

\section{5. $\left.H_{t}\right|_{T^{2} \mid \mathrm{cl} U_{t}}$ has the Bernoulli property}

By the Pesin theory [8], for almost every $z \in T^{2} \mid c l U_{t}$ there exist local unstable and stable manifolds $W_{\text {loc }}^{u}(z), W_{\text {loc }}^{s}(z)$. To prove the Bernoulli property, also by use of the Pesin theory, it is enough to prove that for almost every pair $z, z^{\prime} \in T^{2} \mid \mathrm{cl} U_{t}$, for every $m, n>0$, sufficiently large integers (depending on $z$ and $z^{\prime}$ )

$$
H_{t}^{n}\left(W_{\text {loc }}^{u}(z)\right) \cap H^{-m}\left(W_{\text {loc }}^{s}\left(z^{\prime}\right)\right) \neq \varnothing .
$$

We consider in fact any lifts of these curves and a lift of the dynamics to $\mathbb{R}^{2}$ without any change of notation.

The vectors tangent to the curves $H_{t}^{n}\left(W_{\text {loc }}^{u}(z)\right), H_{t}^{-m}\left(W_{\text {loc }}^{s}\left(z^{\prime}\right)\right)$ lie in the cone bundles $\mathscr{D}$ and $\mathscr{D}^{s}$ respectively, hence the coordinate $x$ is monotonic along these curves, so that we can introduce a natural orientation on those curves and denote the beginning of the curve $H^{n}\left(W_{\text {loc }}^{u}(z)\right)$ by $(x(n, u, b), y(n, u, b))$ and its end by $(x(n, u, e), y(n, u, e))$. Use similar notation for the ends of $H^{-m}\left(W_{\text {loc }}^{s}(z)\right)$ with $u$ replaced by $s$. For almost every $z, z^{\prime}$

$$
\text { length } H_{t}^{n}\left(W_{\text {loc }}^{u}(z)\right) \text {, length } H_{t}^{-m}\left(W_{\text {loc }}^{s}\left(z^{\prime}\right)\right) \underset{m, n \rightarrow \infty}{\longrightarrow} \infty,
$$

hence

$$
|x(n, u, b)-x(n, u, e)| \underset{n \rightarrow \infty}{\longrightarrow} \infty, \quad|x(m, s, b)-x(m, s, e)| \underset{m \rightarrow \infty}{\longrightarrow} \infty
$$


From this it easily follows that

and that

$$
|y(n, u, b)-y(n, u, e)| \underset{n \rightarrow \infty}{\longrightarrow} \infty,|y(m, s, b)-y(m, s, e)| \underset{m \rightarrow \infty}{\longrightarrow} \infty
$$

$$
\frac{x(n, u, b)-x(n, u, e)}{y(n, u, b)-y(n, u, e)}>0, \quad \frac{x(m, s, b)-x(m, s, e)}{y(m, s, b)-y(m, s, e)}<0
$$

for $n, m$ sufficiently large.

This for geometric reasons proves (8).

6. Additional properties of $\left.H_{t}\right|_{T^{2} \backslash \mathrm{cl} U_{t}}$

We begin with the following lemma, where we gather standard facts about the dynamics near a saddle, which we shall need later.

LEMMA 4. Let $0 \in \mathbb{R}^{2}$ be a saddle for a $C^{2}$-diffeomorphism $\phi$ of $\mathbb{R}^{2}$, with eigenvectors $(\partial / \partial x)(0),(\partial / \partial y)(0)$, corresponding eigenvalues $\mu>1, \mu^{-1}$ and stable and unstable manifolds coinciding respectively with the $y$-th and $x$-th axes. Let

$$
\gamma=\left(\gamma_{1}, \gamma_{2}\right):[0,1] \rightarrow \mathbb{R}^{2}
$$

be a $C^{2}$-curve such that

$$
\gamma(0)=0 \quad \text { and } d \gamma_{i} / d s(0)>0 \quad \text { for } i=1,2 .
$$

Let $U$ be a small neighbourhood of 0 . The curve $\gamma$ divides the domain

$$
U^{+}=U \cap\left\{(x, y) \in \mathbb{R}^{2}: x>0, y>0\right\}
$$

into $U_{1}$ whose closure contains an interval from the $y$-axis and $U_{2}$.

Then for every $\delta, C>0$ there exists an integer $m>0$ such that for every

$$
z=\left(x_{0}, y_{0}\right) \in \mathbb{R}^{2}, \quad v \in T_{z} \mathbb{R}^{2}
$$

with the properties:

$$
z, \phi^{N}(z) \notin U, \quad \phi^{n}(z) \in U^{+} \text {for every } n=1, \ldots, N-1
$$

and

$$
\left\|D \phi^{N}(v)\right\| \geq C \cdot\|v\|
$$

the following properties are true:

(a) $\phi^{n}(z) \in U_{1}$ for every $n: 1 \leq n \leq(N / 2)-m$;

(b) $\phi^{n}(z) \in U_{2}$ for $(N / 2)+m \leq n \leq N-1$;

(c) $\left\|D \phi^{n+1}(v)\right\| /\left\|D \phi^{n}(v)\right\|>\mu-\delta$ for $(N / 2)+m \leq n \leq N$;

(d) angle $\left(D \phi^{n}(v), \partial / \partial x\right)<\delta$ for $(N / 2)+m \leq n \leq N$;

(e) If in addition the angle $(v, \partial / \partial y)<C^{-1} x_{0}$, then for $0 \leq n \leq(N /(2+\delta))-m$

$$
\left\|D \phi^{n+1}(v)\right\| /\left\|D \phi^{n}(v)\right\|<\mu^{-1}+\delta \text {. }
$$

We now fix a negative $t$ and study the individual map $H_{t}$.

Proposition 1. The measurable, $D H_{t}$-invariant stable and unstable sub-bundles $E^{s}$ and $E^{u}$, which exist over almost whole $T^{2} \mid \mathrm{cl} U_{t}$ according to Pesin, are actually defined and continuous over the whole $T^{2} \mid \mathrm{cl} U_{t}$. 
Moreover for every $v \in E^{u}, v \neq 0$,

$$
\lim _{n \rightarrow \infty} \inf \frac{1}{n} \log \left\|D H_{t}^{n}(v)\right\|>0
$$

for all neighbourhoods $U_{1}, U_{2}$ of $p_{t}$ and $q_{t}$ respectively, there exists $\delta\left(U_{1}, U_{2}\right)>0$ such that:

$$
\begin{gathered}
\sup \left\{\left\|D H_{t}^{-n}(v)\right\| /\|v\|: n \geq 0, v \in E^{u}(z), v \neq 0,\right. \\
\left.z \in T^{2} \mid \operatorname{cl}\left(U_{t} \cup U_{1} \cup U_{2}\right)\right\}<\delta\left(U_{1}, U_{2}\right)
\end{gathered}
$$

for every $v \in E^{u}$,

$$
\lim _{n \rightarrow \infty}\left\|D H_{t}^{-n}(v)\right\|=0
$$

The analogous properties hold for $E^{s}$. We denote the respective formulae by $\left(9^{s}\right)-\left(11^{s}\right)$.

Proof. We take as $E^{u}$ the line bundle $E_{t}$ described in remark 2, $\$ 3$. Similarly we define $E^{s}$. For every $z \in Q=\operatorname{cl} P\left(C_{1}, 1-C_{1}\right)$,

$$
E^{u}(z) \subset \mathscr{D}_{Q}=\mathscr{D}_{Q}^{u}
$$

(see notation at the end of $\S 4$ ). Clearly for every $z \in Q$,

$$
E^{s}(z) \subset \mathscr{D}_{z}^{s} \subset \mathscr{D}_{Q}^{s}=\left\{(\xi, \eta) \in \mathbb{R}^{2}: \frac{2 \sqrt{|t|}}{1-\sqrt{|t|}} \geq \eta / \xi \geq-1\right\} \text {. }
$$

If $|t|$ is so small that

$$
-2|t| /(1-\sqrt{|t|})+\alpha\left(C_{1}\right)>2 \sqrt{|t|} / /(1-\sqrt{|t|})
$$

then there exist two constant cones of width $\beta>0$ which separate $\mathscr{D}_{Q}^{u}$ and $\mathscr{D}_{Q}^{s}$, hence separate $\left.E^{u}\right|_{Q}$ and $\left.E^{s}\right|_{Q}$ (figure 7 ). So there exists a number $M(\beta)$ such that if $v \in \mathscr{D}_{Q}^{u}$ is decomposed into

$$
v=v_{u}(z)+v_{s}(z)
$$

where

$$
v_{u}(z) \in E^{u}(z), \quad v_{s}(z) \in E^{s}(z) \text { and } z \in Q
$$

then

$$
\left\|v_{s}(z)\right\| /\left\|v_{u}(z)\right\|<M(\beta)
$$

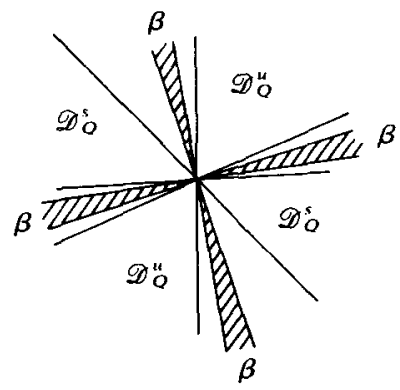

FIGURE 7 
Now assume that $z \notin W^{u}\left(p_{t}\right) \cup W^{u}\left(q_{t}\right)$ (global unstable manifolds). In this case the continuity of $E^{u}$ at $z$ can be proved similarly to the case of Anosov diffeomorphisms. Namely, let us take a constant $C_{2}>C_{1}\left(C_{2} \approx C_{1}\right)$ and denote

$$
Q^{\prime}=P\left(C_{2}, 1-C_{2}\right) \text {. }
$$

Let $i_{1}<i_{2}<\cdots<i_{k}<\cdots$ be all consecutive non-negative integers such that $H_{t}^{-i_{k}}(z) \in Q^{\prime}$. We can consider the continuity of $E^{u}$ at $H_{t}^{-i_{1}}(z)$, i.e. assume that $z \in Q^{\prime}\left(i_{1}=0\right)$.

If $z^{\prime}$ is close to $z$ then $H_{t}^{-i_{k}}\left(z^{\prime}\right)$ is close to $H_{t}^{-i_{k}}(z)$ for $k=1, \ldots, K$ with $K$ large. Hence

$$
H_{t}^{-i_{k}}\left(z^{\prime}\right) \in Q \text {. }
$$

Let $v \in E^{u}\left(z^{\prime}\right)$ and denote $D H_{t}^{-i_{k}}(v)=v_{s}^{k}+v_{u}^{k}$ the decomposition in

$$
E^{s}\left(H_{t}^{-i_{k}}(z)\right) \oplus E^{u}\left(H_{t}^{-i_{k}}(z)\right) \text {. }
$$

Then

$$
\left\|v_{s}^{k}\right\| /\left\|v_{u}^{k}\right\| \leq M_{1} \cdot M(\beta) \cdot\left(\lambda_{t}-\delta\right)^{-2(K-k)}
$$

for small $\delta>0$. We recall from $\S 4$ that $\lambda_{t}>1$ is the constant of hyperbolicity for the differential $D\left(\left(H_{t}\right)_{Q}\right)$ of the first return map $\left(H_{t}\right)_{Q}$. The coefficient $M_{1}$ appears when we pass from the $x$-coordinate ()$_{x}$ used as a norm on $E^{s}$ and $E^{u}$ in $\S 4$ to the norm \|\| . In particular,

$$
\left\|v_{s}^{1}(z)\right\| /\left\|v_{u}^{1}(z)\right\| \leq M_{1} \cdot M(\beta) \cdot\left(\lambda_{t}-\delta\right)^{-2(K-1)}
$$

is small.

In the case $z \in W^{u}\left(p_{t}\right) \cup W^{u}\left(q_{t}\right)$ the continuity of $E^{u}$ in $z$ follows immediately from lemma $4(d)$ and the following lemma.

LEMMA 5. For every $\delta>0$ there exists $C(\delta)>0$ such that if

$$
v \in E^{u}(z), \quad v \neq 0, \quad z \in T^{2} \mid \operatorname{cl} U_{t}
$$

and for $N>0$

$$
H_{t}^{N}(z) \in \operatorname{cl} P(\sqrt{|t|}+\delta, 1-\sqrt{|t|}-\delta)
$$

then

$$
\left\|D H_{t}^{N}(v)\right\| /\|v\|>C(\delta) .
$$

Proof. Let $i_{1}<i_{2}<\cdots$ be the sequence (finite or infinite) of all consecutive nonnegative times when $H_{t}^{i_{k}}(z) \in Q$. We know that for $k=1,2, \ldots$,

$$
\left(D H_{t}^{i_{k+1}}(v)\right)_{x} /\left(D H_{t}^{i_{k}}(v)\right)_{x} \geq \lambda_{t}>1 .
$$

Let $n(z) \geq 0$ be the first time such that

$$
H^{n}(z) \in \operatorname{cl} P(\sqrt{|t|}+\delta, 1-\sqrt{|t|}-\delta)
$$

for every $n: n(z) \leq n \leq i_{1}(z)$. Clearly the set of all possible integers $i_{1}(z)-n(z)$ is bounded from above.

It can happen that $i_{1}(z)$, and consequently $n(z)$, do not exist if $z$ belongs to a component of $W^{s}\left(p_{t}\right) \backslash Q$ or $W^{s}\left(q_{t}\right) \backslash Q$ containing $p_{t}$ or $q_{t}$ respectively. It can also happen that $N<n(z)$ if $\delta<C_{1}-\sqrt{|t|}$. However the set of all possible $N$ in these cases is bounded from above. In the latter case this is due to lemma 1 which implies 
that for every $n: 0<n \leq N$,

$$
H_{t}^{n}(z) \in \operatorname{cl} P\left(-C_{1},-\sqrt{|t|}-\delta\right)
$$

or for every $n: 0<n \leq N$,

$$
H_{t}^{n}(z) \in \operatorname{cl} P\left(\sqrt{|t|}+\delta, C_{1}\right)
$$

The above observations also apply to the point $H^{i_{k}+1}(z)$ where $k$ is the largest integer such that $i_{k}<N$.

Thus, the proof of the lemma reduces to estimating

$$
\left\|D H_{t}^{n(z)}(v)\right\| /\|v\| \text { for } n(z) \text { large. }
$$

Let $z=(x, y)$ and for example $y>0$. Consider the case when

$$
z, H_{t}(z) \in \operatorname{cl} P(0, \sqrt{|t|}) \text {. }
$$

Put

$$
z=z_{0}=\left(x_{0}, y_{0}\right), \quad z_{1}=F_{\text {id }}(z)=\left(x_{1}, y_{0}\right)
$$

and consider the points $z_{i}^{\prime}=\left(x_{i}^{\prime}, y_{i}^{\prime}\right)$ lying on the same vertical as $z_{i}$ belonging to $\gamma_{t}^{+}$, for $i=0$ and to graph $y_{i}^{-}$for $i=1$, see figure 8 .

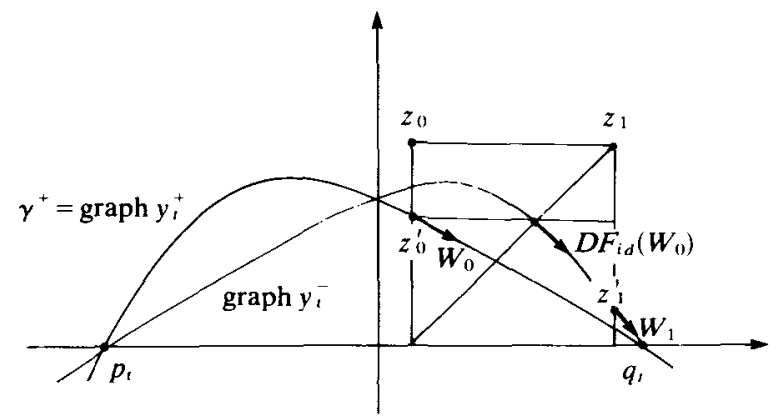

Figure 8

Denote by $W_{0}$ the vector tangent to $\gamma_{t}^{+}$at $z_{0}^{\prime}$ such that $\left(W_{0}\right)_{x}=1$ and by $W_{1}$ the vector tangent to graph $y_{t}^{-}$at $z_{1}^{\prime}$ such that

$$
\left(W_{1}\right)_{x}=\left(D F_{\text {id }}\left(W_{0}\right)\right)_{x} \text {. }
$$

Denote the vectors $W_{i}$ at $z_{i}$ instead of at $z_{i}^{\prime}$ by $W_{i}\left(z_{i}\right)$, for $i=0,1$. Instead of $v$ it is enough to consider $W_{0}\left(z_{0}\right)$.

Put $\left.u=D F_{\text {id }}\left(W_{0}\left(z_{0}\right)\right)-W_{1}\left(z_{1}\right)\right)$. Since

$$
D F_{\text {id }}\left(W_{0}\left(z_{0}\right)\right)=D F_{\text {id }}\left(W_{0}\right)
$$

if we identify the respective tangent spaces, we have

$$
\begin{aligned}
(u)_{y} & =\left(\frac{d y_{t}^{-}}{d x}\left(\left(F_{\mathrm{id}}\left(z_{0}^{\prime}\right)\right)_{x}\right)-\frac{d y_{t}^{-}}{d x}\left(x_{1}\right)\right) \cdot\left(W_{1}\right)_{x} \\
& \geq\left(\sup \left\{\frac{d^{2} y_{t}^{-}}{d x^{2}}(x): x \in[-\sqrt{|t|}, \sqrt{|t|}]\right\}\right) \cdot\left(y_{0}^{\prime}-y_{0}\right) \cdot\left(W_{1}\right)_{x} \\
& >\frac{3}{2} \cdot\left(y_{0}-y_{0}^{\prime}\right) \cdot(1-\sqrt{|t|}) /(1+\sqrt{|t|})>y_{0}-y_{1}^{\prime} .
\end{aligned}
$$

Of course $(u)_{x}=0$. 
Let $I:[0,1] \rightarrow T^{2}$ be the interval, joining $z_{1}^{\prime}$ with $z_{1}$. Using the convexity of the function $g_{t}$ in the domain $\left[0, \frac{1}{4}\right]$ one can prove by induction that for every $s \in[0,1]$ and $n: 0 \leq n \leq n(z)-1$ :

$$
\left(D\left(H_{t}^{n} \circ G_{g_{t}}\right)(u)\right)_{x} \geq\left(D\left(H_{t}^{n} \circ G_{\mathbf{g}_{t}} \circ I\right)((\partial / \partial s)(s))\right)_{x} .
$$

Since

$$
D\left(H_{t}^{n} \circ G_{g_{t}}\right)\left(W_{1}\left(z_{1}\right)\right), D\left(H_{t}^{n} \circ G_{\mathbf{g}_{t}}\right)(u) \in \mathscr{D} \quad \text { for } n \geq 1,
$$

we have

$$
\left(D\left(H_{t}^{n} \circ G_{g_{t}}\right)\left(W_{1}\left(z_{1}\right)\right)\right)_{x},\left(D\left(H_{t}^{n} \circ G_{g_{t}}\right)(u)\right)_{x}>0 .
$$

So

$$
\begin{aligned}
\left(D H_{t}^{n(z)}\left(W_{0}\left(z_{0}\right)\right)_{x} \geq\right. & \int_{0}^{1}\left(D\left(H_{t}^{n(z)-1} \circ G_{\mathbf{g}_{t}} \circ I\right)((\partial / \partial s)(s))\right)_{x} d s \\
& +\left(D\left(H_{t}^{n(z)-1} \circ G_{\mathbf{g}_{t}}\right)\left(W_{1}\left(z_{1}\right)\right)\right)_{x}>\delta,
\end{aligned}
$$

since $\left(H_{t}^{n(z)-1} G_{\mathbf{g}_{t}}\right)\left(z_{1}^{\prime}\right)$ stays in $\gamma_{t}^{+}$, hence in $P(-\sqrt{|t|}, \sqrt{|t|})$ and

$$
\left(H_{t}^{n(z)-1} G_{g_{t}}\right)\left(z_{1}\right) \in P(\sqrt{|t|}+\delta, 1-\sqrt{|t|}-\delta) .
$$

We have considered the case $z, H_{t}(z) \in \operatorname{cl} P(0, \sqrt{|t|})$.

The case $z \in P\left(-C_{1}, 0\right)$ reduces to the previous one since

$$
\left(D H_{t}^{n+1}(v)\right)_{x} /\left(D H_{t}^{n}(v)\right)_{x} \geq 1 \text { for every } n=0,1, \ldots, n_{1}(z)-2,
$$

where $n=n_{1}(z)$ is the first time when

$$
H_{t}^{n}(z) \in \operatorname{cl} P(0, \sqrt{|t|}) .
$$

Then also

$$
H_{t}^{n_{1}(z)+1}(z) \in \operatorname{cl} P(0, \sqrt{|t|})
$$

due to the assumption that $n(z)$ is large.

Also for $z \in \mathscr{T}_{t}\left(C_{1}-\sqrt{|t|}\right) \cup \mathscr{T}_{t}^{\prime}\left(C_{1}-\sqrt{|t|}\right)$ we have

$$
\left(D H_{t}^{n+1}(v)\right)_{x} /\left(D H_{t}^{n}(v)\right)_{x}>1 \text { for every } n=0,1, \ldots, n(z)-1 .
$$

The less trivial case is when $z \in P(\sqrt{|t|}, \sqrt{|t|}+\delta)$. We still assume that $y>0$ :

Let $n=n^{\prime}(z)>0$ be the first time that

$$
H_{t}^{-n}(z) \in \operatorname{cl} P(0, \sqrt{|t|}-\delta) .
$$

Notice that it is enough to prove the lemma only for $\delta \ll \sqrt{|t|}$. Now we shall use lemma 4 for the saddle $q_{t}$, its neighbourhood: the square with the sides $x=\sqrt{|t|} \pm \delta$ and $y= \pm \delta$ and for the curve $\{x=\sqrt{|t|}, y \geq 0\}$. For that we need to change coordinates. Its assumptions, for the vector $D H_{t}^{-n^{\prime}(z)}(v)$ tangent of $H_{t}^{-n^{\prime}(z)}(z)$ are satisfied due to the proved case of lemma 5 .

So, by lemma 4(c)

$$
\left\|D H_{t}^{n+1}(v)\right\|^{\prime} /\left\|D H_{t}^{n}(v)\right\|^{\prime} \geq 1
$$

for every $n$ such that

$$
\left(n(z)+n^{\prime}(z)\right) / 2+m-n^{\prime}(z) \leq n \leq n(z) .
$$


Here we use the Euclidean norm \|\|$^{\prime}$ connected with the coordinates of lemma 4.

By lemma $4(a)$, since $z \in P(\sqrt{|t|}, \sqrt{|t|}+\delta)$

$$
n^{\prime}(z) \geq \frac{n(z)+n^{\prime}(z)}{2}-m \text {. }
$$

Hence (12) holds for every $n=2 m, \ldots, n(z)-1$. So

$$
\left\|D H_{t}^{n(z)}(v)\right\| /\|v\| \geq C L^{-2 m}
$$

where $L$ is the Lipschitz constant for $H_{t}^{-1}$ and $C$ is a coefficient connected with the change of the norms. This ends the proof of lemma 5.

We still need to prove $\left(9^{u(s)}\right)-\left(11^{u(s)}\right)$ in proposition 1 . Let us start with $\left(9^{u}\right)$. This is obvious for

$$
z \in W^{s}\left(p_{t}\right) \cup W^{s}\left(q_{t}\right) .
$$

To prove the other case it is enough to find $\mu_{t}>1$ such that for every $z \in Q$, if the first positive integer $n(z)$ such that $H_{t}^{n(z)} \in Q$ is larger than a constant integer $N$,

$$
\left(D H_{t}^{n}(v)\right)_{x} /(v)_{x} \geq \mu_{t}^{n} \quad \text { for } n=0,1, \ldots, n(z) .
$$

Then we would obtain in $\left(9^{u}\right)$ the estimate by

$$
\min \left(N^{-1} \log \lambda_{t}, \log \mu_{t}\right) \text {. }
$$

Let $z=(x, y) \in Q, n(z)$ be as above with $y>0$. Let $n=n_{1}(z)$ be the first positive integer such that

$$
H_{\imath}^{n}(z) \in P\left(0, C_{1}\right)
$$

We extend the notation from the proof of lemma $3, \S 4$ : For every $n=0,1, \ldots, n(z)$ put

$$
R(n)=\left(D H_{t}^{n}(v)\right)_{x} /(v)_{x}=\prod_{k=0}^{n-1} l_{k}
$$

where $l_{n}=\left(D H_{t}^{n+1}(v)_{x} /\left(D H_{t}^{n}(v)\right)_{x}\right.$. Put as usual $H_{t}^{n}(z)=\left(x_{n}, y_{n}\right)$ and, furthermore:

$r_{n}=1-2 \sqrt{|t|} /(1-\sqrt{|t|})+\alpha\left(C_{1}\right)$ for $n=0$

$r_{n}=(1+\sqrt{|t|}) /(1-\sqrt{|t|})$ for $n \geq 0$ and such that $x_{n}<-\sqrt{|t|}$;

$r_{n}=1+d y_{t}^{+} / d x\left(x_{n}\right)$ if $-\sqrt{|t|} \leq x_{n}$ and $n \leq n_{1}(z)-2$ and also for $n=n_{1}(z)-1$ we assume that $\left|x_{n_{1}(z)-1}\right|>\left|x_{n_{1}(z)}\right|$;

$$
r_{n}=\left(1-d y_{t}^{-} / d x\left(x_{n+1}\right)\right)^{-1} \text { if } x_{n+1} \leq \sqrt{|t|} \text { and } n \geq n_{1}(z)
$$

and also for $n=n_{1}(z)-1$ we assume that $\left|x_{n_{1}(z)-1}\right| \leq\left|x_{n_{1}(z)}\right|$;

$$
r_{n}=(1-\sqrt{|t|}) /(1+\sqrt{|t|}) \text { if } x_{n+1}>\sqrt{|t|} \text { and } n<n(z) \text {. }
$$

Recall that $l_{n} \geq r_{n}$. Due to lemma 5 we can use lemma $4(b)$ and $(c)$, so there exists $m>0$ such that for every $n$ satisfying:

$$
n_{4}(z)=n_{1}(z)+\left(n(z)-n_{1}(z)\right) / 2+m \leq n \leq n(z)
$$

we have

$$
x_{n}>\sqrt{|t|} \quad \text { and } \quad l_{n}>((1+\sqrt{|t|}) /(1-\sqrt{|t|}))^{\frac{1}{2}}
$$


So

$$
\begin{aligned}
R_{n_{4}(z)} & =\prod_{i=0}^{n_{4}(z)-1} l_{i} \geq\left(\prod_{i=0}^{n(z)-1} r_{i}\right) \cdot\left(\prod_{i=n_{4}(z)}^{n(z)-1} r_{i}\right)^{-1} \\
& \geq \lambda_{t} \cdot\left(\frac{1-\sqrt{|t|} \mid}{1+\sqrt{|t|}}\right)^{-n(z) / 5} \geq\left(\left(\frac{1+\sqrt{|t|} \mid}{1-\sqrt{|t|}}\right)^{\frac{1}{5}}\right)^{n_{4}(z)} .
\end{aligned}
$$

We have used the fact that for large $n(z), n_{4}(z)<{ }_{5}^{4} n(z)$. This is true due to the definition of $n_{4}(z)$ and due to lemma $2, \S 4$ which gives $\left|n_{1}(z)-n(z) / 2\right| \leq 1$.

For $n \geq n_{4}(z)$, we have due to (13):

$$
\begin{aligned}
R(n) & =R\left(n_{4}(z)\right) \cdot\left(\prod_{i=n_{4}(z)}^{n-1} l_{i}\right) \\
& \geq\left(\left(\frac{1+\sqrt{|t|}}{1-\sqrt{|t|}}\right)^{\frac{1}{5}}\right)^{n_{4}(z)}\left(\left(\frac{1+\sqrt{|t|}}{1-\sqrt{|t|}}\right)^{\frac{1}{2}}\right)^{n-n_{4}(z)}>\left(\left(\frac{1+\sqrt{|t|} \mid}{1-\sqrt{|t|} \mid}\right)^{\frac{1}{5}}\right)^{n} .
\end{aligned}
$$

For $n \leq n_{4}(z)$ similar estimates follow from

$$
R(n) \geq \prod_{i=0}^{n-1} r_{i}, \quad \prod_{i=0}^{n_{4}(z)-1} r_{i} \geq\left(\left(\frac{1+\sqrt{|t|}}{1-\sqrt{|t|}}\right)^{\frac{1}{5}}\right)^{n_{4}(z)}
$$

and from the fact that the sequence $r_{i}, i=0, \ldots, n_{4}(z)$ is decreasing.

Concluding, we can take

$$
\mu_{t}=\left((1+\sqrt{|t|}) /(1-\sqrt{|t|} \mid)^{\frac{1}{5}} .\right.
$$

A more careful estimate in (14) would show that we could take

for arbitrarily small $\delta>0$.

$$
\mu_{t}=((1+\sqrt{|t|}) /(1-\sqrt{|t|}))^{\frac{1}{3}-\delta}
$$

Now let us prove $\left(10^{u}\right)$. Let $U_{1}, U_{2}$ contain respectively some balls $B\left(p_{t}, \delta\right)$, $B\left(q_{t}, \delta\right)$. For

$$
z \in \operatorname{cl} P(\sqrt{|t|}+\delta / 2,1-\sqrt{|t|}-\delta / 2)
$$

$\left(10^{u}\right)$ follows from lemma 5 . If

$$
z \in \operatorname{cl} P(-\sqrt{|t|}-\delta / 2, \sqrt{|t|}+\delta / 2),
$$

then $z$ is within the distance of at least $\delta / 2$ from the components $W^{p}$ and $W^{a}$ of

$$
W^{u}\left(p_{t}\right) \cap \operatorname{cl} P(-\sqrt{|t|}-\delta / 2,-\sqrt{|t|})
$$

or

$$
W^{u}\left(q_{t}\right) \cap \operatorname{cl} P(\sqrt{|t|}, \sqrt{|t|}+\delta / 2)
$$

containing $p_{t}$ or $q_{t}$, respectively, since $W^{p}$ and $W^{q}$ are almost horizontal if $|t|$ is sufficiently small. So after bounded time $n>0$ and some time $m \geqslant 0$ during which $D H_{t}^{-1}$ contracts on $E^{u}$,

$$
H_{t}^{-n}(z) \in \operatorname{cl} P(\sqrt{|t|}+\delta / 2,1-\sqrt{|t|}-\delta / 2)
$$

and we have the previous case. 
$\left(11^{u}\right)$ is obvious in the case $z \in W^{u}\left(p_{t}\right) \cup W^{u}\left(q_{t}\right)$. In the other case it follows from $\left(10^{u}\right)$ and from the fact that

$$
\left(D H_{t}^{-i_{k+1}}(v)\right)_{x} /\left(D H_{t}^{-i_{k}}(v)\right)_{x} \leq \lambda_{t}^{-1}
$$

for every two consecutive times $i_{k}, i_{k+1}$ when the backward trajectory $H_{t}^{-n}(z)$ of $z$ hits $Q$. The proof of proposition 1 is finished.

COROLlARY 1 . For every $H_{t}$-invariant probability measure on $T^{2} \mid \mathrm{cl} U_{t}$, the Lyapunov characteristic exponents are almost everywhere non-zero and of opposite signs.

Proof. This corollary follows from $\left(9^{u}\right)$ and $\left(9^{s}\right)$.

COROllaRy 2. For every $\delta>0$ there exists $C_{0}(\delta)>0$ such that for every $\gamma^{u}:[0,1] \rightarrow T^{2} \backslash \mathrm{cl} U_{t}$ an integral curve for $E^{u}$, if

$$
\operatorname{dist}\left(\gamma^{\prime \prime},\left\{p_{t}\right\} \cup\left\{q_{t}\right\}\right) \geq \delta
$$

then

$$
\sup _{n \geq 0}\left(\text { length } H_{t}^{-n}\left(\gamma^{u}\right) / \text { length } \gamma^{u}\right) \leq C_{0}(\delta)
$$

and

$$
\lim _{n \rightarrow \infty} \text { length } H_{t}^{-n}\left(\gamma^{u}\right)=0
$$

The analogous facts hold for integral curves for $E^{s}$.

Proof.

$$
\begin{aligned}
\lim _{n \rightarrow \infty} \text { length } H_{t}^{-n}\left(\gamma^{u}\right) & =\lim _{n \rightarrow \infty} \int_{0}^{1}\left\|D\left(H_{t}^{-n} \circ \gamma^{u}\right)(\partial / \partial s)(s)\right\| d s \\
& =\int_{0}^{1}\left(\lim _{n \rightarrow \infty} \| D\left(H_{t}^{-n} \circ \gamma^{u}\right)(\partial / \partial s)(s)\right) \| d s=0 .
\end{aligned}
$$

We used the fact that the integrands are uniformly bounded by $\left(10^{\mu}\right)$ and converge to 0 pointwise by $\left(11^{u}\right) .\left(10^{u}\right)$ gives $(15)$ with

$$
C_{0}(\delta)=C\left(B\left(p_{t}, \delta\right), B\left(q_{t}, \delta\right)\right) \text {. }
$$

Let $z=\left(x_{0}, y_{0}\right) \in T^{2} \backslash \mathrm{cl} U_{t}$. Consider the rectangle

$$
S=\left\{(x, y): x_{0}-\delta \leq x \leq x_{0}+\delta, y_{0}-K \delta \leq y \leq y_{0}+K \delta\right\},
$$

where $K=1+\sup d g_{t} / d x$ and $\delta$ such that $S \cap \operatorname{cl} U_{t}=\varnothing$ and

$$
(K+1) \cdot \delta \cdot C_{0}\left(\operatorname{dist}\left(S,\left\{p_{t}\right\} \cup\left\{q_{t}\right\}\right)\right) \ll 1 .
$$

Let $\gamma^{u} \ni z$ be a maximal integral curve for $E^{u}$ in $S$. By the definition of $K$ it joins the left and right hand sides of $S$. Then $\gamma^{u}$, the candidate for a local unstable manifold has the following characterization:

$$
\begin{aligned}
\gamma^{u}= & \left\{z^{\prime} \in S: \operatorname{dist}\left(H_{t}^{-n}\left(z^{\prime}\right), H_{t}^{-n}(z)\right) \leq \operatorname{dist}\left(z^{\prime}, z\right) \cdot(K+1)\right. \\
& \left.\times C_{0}\left(\operatorname{dist}\left(S,\left\{p_{t}\right\} \cup\left\{q_{t}\right\}\right)\right) \text { for every } n \geq 0\right\} .
\end{aligned}
$$


The inclusion ' $C$ ' follows from (15) in corollary 2. To prove ' $\supset$ ' take

$$
u=\left(x_{1}, y_{1}\right) \in S \backslash \gamma^{u}
$$

and put $u^{\prime}=\left(x_{1}, y_{1}^{\prime}\right)$ the point on the same vertical as $u$, in $\gamma^{u}$. Take the interval $I$ joining $u$ with $u^{\prime}$. For every $n \geq 2$ the vectors tangent to $H_{t}^{-n}(I)$ belong to the stable cones $\mathscr{D}^{s}$. Hence

$$
\begin{aligned}
\sup _{n \geq 0} \operatorname{dist}\left(H_{t}^{-n}(u), H_{t}^{-n}(z)\right) \geq & \sup _{n \geq 0} \operatorname{dist}\left(H_{t}^{-n}(u), H_{t}^{-n}\left(u^{\prime}\right)\right) \\
& -\sup _{n \geq 0} \operatorname{dist}\left(H_{t}^{-n}\left(u^{\prime}\right), H_{t}^{-n}(z)\right) \\
\geq & \frac{1}{2} L^{-1}-(K+1) \cdot \delta \cdot C_{0}\left(\operatorname{dist}\left(S,\left\{p_{t}\right\} \cup\left\{q_{t}\right\}\right)\right) \geq \text { const }>0 .
\end{aligned}
$$

$L$ is the Lipschitz constant for $H_{t}^{-1}$.

The above characterization of $\gamma^{u}$ and an analogous characterization of $\gamma^{s}$ prove:

COROLlary 3. The line bundles $E^{u}$ and $E^{s}$ are uniquely integrable.

Remark. The bundles $E^{u}$ and $E^{s}$ extend to the continuous bundles $\bar{E}^{u}$ and $\bar{E}^{s}$ over $T^{2} \backslash\left(U_{t} \cup\left\{p_{t}\right\} \cup\left\{q_{t}\right\}\right)$ which are tangent to $\gamma_{t}^{ \pm}$over $\gamma_{t}^{ \pm}$. It is easy to see that $\left(10^{u(s)}\right),\left(11^{u(s)}\right)$, corollary 2 and corollary 3 hold if $E^{u(s)}$ is replaced by $\bar{E}^{u(s)}$.

Proposition 2. There exists a continuous semiconjugacy $\varphi: T^{2} \rightarrow{ }^{\text {onto }} T^{2}$ from $H_{t}$ to the Anosov automorphism $A$ (i.e. $\varphi \circ H_{t}=A \circ \varphi$ ) such that $\varphi^{-1}(0)=\operatorname{cl} U_{t}$ and $\left.\varphi\right|_{T^{2} \mid \mathrm{cl}} U_{t}$ is 1-1. This means that $\left.H_{t}\right|_{T^{2} \mid \mathrm{cl} U_{t}}$ is topologically conjugated with $\left.A\right|_{T^{2} \backslash\{0\}}$.

Compare this proposition with property (1) of $H_{t}, t \geqslant 0$, from the introduction. Proof. The existence of a semiconjugacy follows from [3, proposition 2.1].

Denote by $\tilde{\varphi}, \tilde{H}, \tilde{A}$ lifts of $\varphi, H_{t}, A$ to $\mathbb{R}^{2}$ keeping $0 \in \mathbb{R}^{2}$ invariant, such that

$$
\tilde{\varphi} \circ \tilde{H}=\tilde{A} \circ \tilde{\varphi} .
$$

$\tilde{\varphi}$-id is a bounded function and $\tilde{A}$ is expansive in the following sense:

$$
\sup _{n \in Z} \operatorname{dist}\left(\tilde{A}^{n}(z), \tilde{A}^{n}\left(z^{\prime}\right)\right)=\infty
$$

for every $z, z^{\prime} \in \mathbb{R}^{2}, z \neq z^{\prime}$.

Hence $\tilde{\varphi}(z) \neq \tilde{\varphi}\left(z^{\prime}\right)$ is equivalent to

$$
\sup _{n \in Z} \operatorname{dist}\left(\tilde{H}^{n}(z), \tilde{H}^{n}\left(z^{\prime}\right)\right)=\infty .
$$

Denote by $\Pi$ the projection $\Pi: \mathbb{R}^{2} \rightarrow \mathbb{R}^{2} / Z^{2}=T^{2}$. Then

$$
\varphi(\Pi(z)) \neq \varphi\left(\Pi\left(z^{\prime}\right)\right)
$$

is equivalent to (16) for every pair $z+w, z^{\prime}$ where $w \in Z^{2}$.

Due to this criterion we immediately have $\varphi\left(\mathrm{cl} U_{t}\right)=0$. To finish the proof it is enough to check that for every pair $z \in \Pi^{-1}\left(T^{2} \mid \mathrm{cl} U_{t}\right), z^{\prime} \in \Pi^{-1}\left(T^{2} \backslash U_{t}\right)$, we have

$$
\sup _{n \in Z} \operatorname{dist}\left(\tilde{H}^{n}(z), \tilde{H}^{n}\left(z^{\prime}\right)\right)=\infty .
$$

We shall only check the case when $z=z_{0}=\left(x_{0}, y_{0}\right)$ and $z^{\prime}=\left(x^{\prime}, y^{\prime}\right)$ are close to 0 and $y_{0}, y^{\prime}>0$ and leave the other cases to the reader. 
Consider the new coordinates $x, \beta(x, y)=y-y_{t}^{+}(x, y)$ in a neighbourhood $W$ of 0 . Put

and

$$
V_{1}=\left\{z=(x, y) \in W:\left(x-x_{0}\right) \cdot\left(\beta(z)-\beta\left(z_{0}\right)\right) \geq 0\right\}
$$

See figure 9.

$$
V_{2}=W \backslash V_{1}
$$

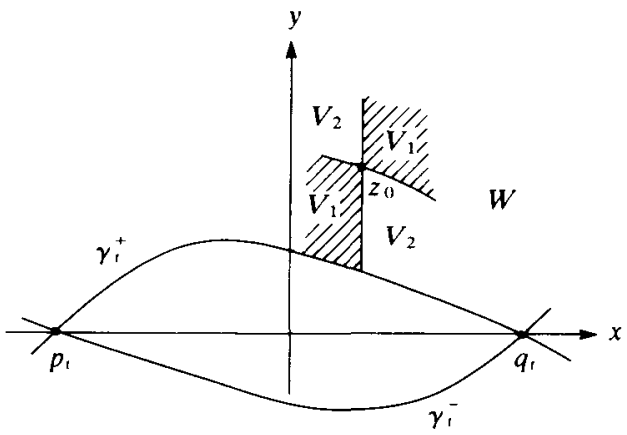

Figure 9

Join $z=z_{0}$ with $z^{\prime}$ by the interval $I:[0,1] \rightarrow \mathbb{R}^{2}$ in the coordinates $(x, \beta)$. We lift our $D H_{t}\left(D H_{t}^{-1}\right)$-invariant cones and bundles $E^{u(s)}$ to $T\left(\mathbb{R}^{2}\right)$ and use the same notation for them as in $T\left(T^{2}\right)$. Now if $z^{\prime} \in V_{1}$

$$
D\left(\tilde{H}^{n} \circ I\right)(\partial / \partial s)(s) \in \mathscr{D}
$$

for every $n=1,2, \ldots$ and $s \in[0,1]$ except maybe $s=s_{0}$ such that $I\left(s_{0}\right) \in \gamma_{t}^{+}$where $\mathscr{D}$ has not been defined. There exists at most one such $s_{0}$ since $z$ and $z^{\prime}$ do not both belong to $\gamma_{t}^{+}$. Hence

$$
D\left(\tilde{H}^{n} \circ I\right)(\partial / \partial s)(s) \notin \bar{E}^{s} .
$$

This is true for $s \neq s_{0}$ since

$$
\mathscr{D} \cap \operatorname{int} \mathscr{D}^{s}=\varnothing
$$

and in fact $E^{s} \subset$ int $\mathscr{D}^{s}$.

For every $s \neq s_{0}$ decompose

$$
D(\tilde{H} \circ I)(\partial / \partial s)(s)=v_{1}(s)+v_{2}(s)
$$

according to the decomposition $E^{s} \oplus E^{u}$. We have the function $\left\|v_{1}(s)\right\|$ bounded from above on $[0,1] \backslash J$ where $J$ is a neighbourhood of $s_{0}$ and also $\left\|v_{2}(s)\right\|>0$ for every $s \in[0,1] \backslash J$.

Then by $\left(9^{u}\right)-\left(11^{u}\right)$

$$
\text { length }\left(\left.\tilde{H}^{n} \circ I\right|_{[0,1] \backslash J}\right) \underset{n \rightarrow \infty}{\longrightarrow} \infty
$$

so length $\left(\tilde{H}^{n} \circ I\right) \rightarrow_{n \rightarrow \infty} \infty$.

Since by $(17)$ the functions $\left(D \tilde{H}^{n}(\partial / \partial s)(s)\right)_{x}$ have constant signs and

$$
\left(D \tilde{H}^{n}(\partial / \partial s)(s)\right)_{y} /\left(D \tilde{H}^{n}(\partial / \partial s)(s)\right)_{x} \leq 1+\sup d g_{t} / d x
$$

is uniformly bounded,

$$
\operatorname{dist}\left(\tilde{H}^{n}(z), \tilde{H}^{n}\left(z^{\prime}\right)\right) \underset{n \rightarrow \infty}{\longrightarrow} \infty
$$


The proof for $z^{\prime} \in V_{2}$ is similar. In that case expansiveness occurs under backward iterates. The proof of proposition 2 is finished.

It occurs that $\left.\varphi\right|_{T^{2} \mid \mathrm{cl} U_{t}}$ cannot be $C^{1}$. Moreover, we shall prove the following proposition.

Proposition 3. There exist no $C^{1}$-diffeomorphisms

$$
B: T^{2} \rightarrow T^{2} \text { and } \varphi:\left(T^{2} \mid \mathrm{cl} U_{t}\right) \rightarrow T^{2} \backslash\{0\}
$$

such that $\varphi \circ H_{t}=B \circ \varphi$.

Proof. We use the method used by Gerber and Katok [4] to prove the analogous fact for pseudo-Anosov homeomorphisms. Due to proposition 2 we can find a Markov partition for $\left.H_{t}\right|_{T^{2} \mid \mathrm{cl}} U_{t}$ containing the cells $M_{i}, i=1,2$ being closures of a neighbourhood of cl $U_{t}$ intersected with $\mathscr{T}_{t}$ and $\{y>0\} \mid\left(\mathscr{T}_{t} \cup \mathscr{T}_{t}^{\prime}\right)$ respectively. So there exist sequences of $H_{t}$-periodic points $z_{n}, w_{n}$ with periods $\alpha_{n}, \beta_{n} \rightarrow \infty$ such that

$$
z_{n} \in \operatorname{int} M_{1}, \quad w_{n} \in \operatorname{int} M_{2}, z_{n}, w_{n} \underset{n \rightarrow \infty}{\longrightarrow} D,
$$

which is a fundamental domain in $W^{s}\left(p_{t}\right)$, and there exists a constant integer $N>0$ such that for every $i, n: 0 \leq i \leq \alpha_{n}-N$,

$$
H^{i}\left(z_{n}\right) \in M_{1}
$$

and for every $i, n: 0 \leq i \leq \beta_{n}-N$,

$$
H^{i}\left(w_{n}\right) \in M_{2}
$$

(see figure 10).

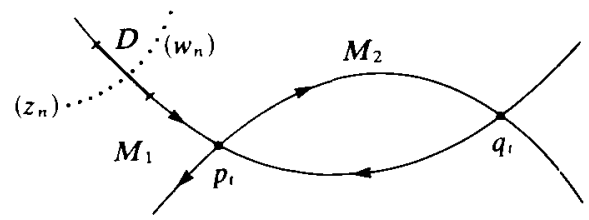

FIGURE 10

Clearly the Lyapunov exponents $\lambda_{ \pm}\left(z_{n}\right)$ converge to the logarithms of the eigenvalues at $p_{t}$, i.e. to

$$
\lambda_{ \pm}^{(z)}= \pm \log (1+\sqrt{|t|}) /(1-\sqrt{|t|})
$$

For $v \in E^{u}\left(w_{n}\right)$ we have clearly

$$
\frac{1}{n_{1}\left(w_{n}\right)} \log \left\|D H_{t}^{n_{1}\left(w_{n}\right)}\left(v_{n}\right)\right\| \approx \log \left(\frac{1+\sqrt{|t|}}{1-\sqrt{|t|}}\right)
$$

for $n$ large, where $n_{1}\left(w_{n}\right)$ is the first time when

$$
\left(H_{t}^{n_{1}\left(w_{n}\right)}\left(w_{n}\right)\right)_{x}>0 .
$$

The bundle $\bar{E}^{u}$ is Lipschitz continuous at $\gamma^{ \pm}$. This is a property of dynamics around the saddle $p_{t}$, compare with [5, theorem 6.3.b]. 
Hence we can use lemma 4(e) for a neighbourhood of $q_{t}$ and conclude by use of lemma $4(c)$ that

$$
\log \left(\left\|D H_{t}^{\beta_{n}-N}\left(v_{n}\right)\right\| /\left\|D H_{t}^{n_{1}\left(w_{n}\right)}\left(v_{n}\right)\right\| \approx 0\right.
$$

for $n$ large.

By lemma $2\left|n_{1}\left(w_{n}\right)-\beta_{n} / 2\right|$ is uniformly bounded for all $n$. So, the Lyapunov exponents $\lambda_{ \pm}\left(w_{n}\right)$ converge to

$$
\lambda_{ \pm}^{(w)}= \pm \frac{1}{2} \log ((1+\sqrt{|t|}) /(1-\sqrt{|t|})) .
$$

If $\varphi$ and $B$ existed, the Lyapunov exponents over $\varphi\left(z_{n}\right)$ and $\varphi\left(w_{n}\right)$ would also converge to $\lambda_{ \pm}^{(z)}, \lambda_{ \pm}^{(w)}$ respectively.

Meanwhile, if one of the eigenvalues of $D B(0)$ were 0 , then

$$
\lim _{n \rightarrow \infty} \frac{1}{n} \log \left\|D B_{\varphi\left(z_{n}\right)}^{\alpha_{n}}\right\|=0 \quad \text { or } \quad \lim _{n \rightarrow \infty} \frac{1}{n} \log \left\|D B_{\varphi\left(z_{n}\right)}^{-\alpha_{n}}\right\|=0,
$$

so $\log \lambda_{+}^{(z)}$ and $\log \lambda_{-}^{(z)}$ could not have different signs. This is a contradiction.

If 0 were a saddle for $\boldsymbol{B}$ then $\varphi\left(z_{n}\right), \varphi\left(w_{n}\right) \rightarrow_{n \rightarrow \infty} \varphi(D)$ - a fundamental domain in a local stable manifold for $B$ at 0 . But the set of limit spaces of the sequences $D \varphi\left(E^{u}\left(z_{n}\right)\right)$ and $D\left(E^{u}\left(w_{n}\right)\right)$ is disjoint with the bundle tangent to $\varphi(D)$. Hence one of the Lyapunov exponents at $\varphi\left(z_{n}\right)$ and at $\varphi\left(w_{n}\right)$ converge to the same number, to the logarithm of an eigenvalue of $D B(0)$. So $\lambda_{+}^{(z)}=\lambda_{+}^{(w)}$. This is a contradiction.

\section{Final remarks}

Remark 1 . We do not know whether there exists a family $g_{t}$ satisfying property (1) $\S 1$, with separatrices joining $p_{t}$ with $q_{t}$ for the corresponding $H_{t}$, such that $g_{t}$ on $\mathbb{R}$, and hence, $H_{t}$ on $T^{2}$, is real-analytic.

The problem is to solve the system of functional equations:

$$
y_{t}^{-}\left(x+y_{t}^{+}(x)\right)=y_{t}^{+}(x) \quad g_{t}=y_{t}^{+}-y_{t}^{-}
$$

close to $t=0, x=0$, in real-analytic functions satisfying property (1) (its part, at $t=x=0$ ), so that $g_{t}-\mathrm{id}$ is periodic with period 1 .

The periodicity condition does not hold for $g_{t}$ defined by $(*)$ in the theorem in $\S 2$. There, the functions $g_{t}$ have real poles.

We can attempt to solve the problem by starting with the family of the Hamiltonian functions:

$$
h_{t}(x, y)=\left(\frac{4}{5} x^{2}-(y+\sqrt{C}-t)^{2}+C\right) \cdot\left(\frac{4}{5} x^{2}-(y-\sqrt{C}+t)^{2}+C\right),
$$

for a constant $C>0$.

Then we obtain $g_{t}-$ id bounded (not periodic unfortunately).

We have chosen the above $h_{t}$ so that the set of their zeros consists of branches of hyperboles. The choice is motivated by the fact that if we want $g_{t}$ to be real-analytic, then graph $y_{t}^{+}$must coincide with the unstable manifold of $q_{t}$ for $H_{t}$ (when $x \rightarrow+\infty$ ). So, when $x \rightarrow+\infty$, graph $y_{t}^{+}$must be within the finite distance from the unstable manifold of 0 for the Anosov diffeomorphism $A$, which is the straight line $(2 x / \sqrt{5})-y=0$. This is so because of the existence of a semiconjugacy from $H_{t}$ to $A$, see proposition $3, \S 6$. 
Remark 2. We could consider directly the time-one diffeomorphism $\bar{H}_{t, 1}$ for the Hamiltonian vector field corresponding to the function

$$
h_{t}(x, y)=y^{2}-x^{2}\left(x^{2}+2 t\right) \text {, }
$$

see $\S 2$. The trouble then is with a simple extension of this diffeomorphism from a neighbourhood of $\operatorname{cl} U_{t}$ to the whole $T^{2}$. Such $\bar{H}_{t, 1}$ on $U_{t}$ would be integrable (i.e. $U_{t} \backslash\{0\}$ would consist of closed invariant curves).

Our $H_{t}$ 's, close to 0 , are perturbations of such $\bar{H}_{t, 1}$. The intuition to treat $H_{t}$ as a time-one solution for a differential equation has been basic to the existence of invariant cones (such a cone cannot pass to the other side of the trajectory of the flow, figure 11) and in lemma 2.

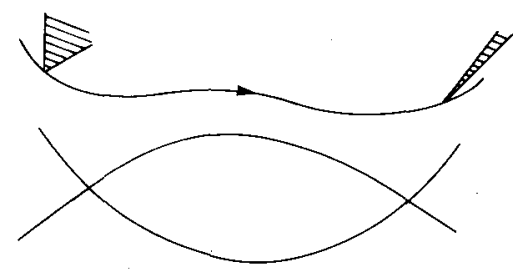

FigURE 11

Remark 3. In the proof of proposition $3 \S 6$ we used the fact that in the construction of $H_{t}, t<0$ only two out of four sectors between stable and unstable manifolds of a saddle of an Anosov diffeomorphism were 'blown up'.

We can however use the Hamiltonian function:

$$
y^{2}\left(y^{2}+2 t\right)-x^{2}\left(x^{2}+2 t\right)=\left(y^{2}-x^{2}\right)\left(x^{2}+y^{2}+2 t\right) .
$$

For $t<0$, the separatrices $\gamma_{i}, i=1, \ldots, 4$, joining the saddles $p_{i}=( \pm \sqrt{|t|}, \pm \sqrt{|t|})$, $i=1, \ldots, 4$, form a circle $S_{t}$, see figure 12 .

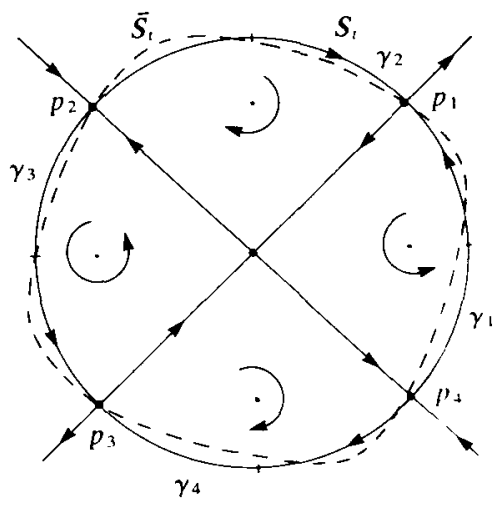

FIGURE 12

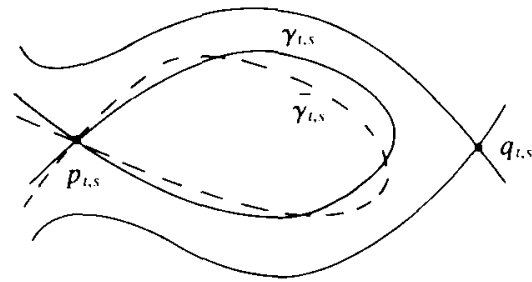

FIGURE 13

Now we should either somehow extend the time-one diffeomorphism for the resulting Hamiltonian vector field or find functions $f=f_{t}, g=g_{v}, \mathbb{R} \rightarrow \mathbb{R}$ with property (1) $\& 1$ such that the toral linked twist mapping $H_{f, g}$ still preserves the saddles $\boldsymbol{p}_{i}$ and a closed curve $\bar{S}_{t}$, built from separatrices $\bar{\gamma}_{i}, i=1, \ldots, 4$ (close to $S_{t}$ ). 
For each individual $t$ it is easy to find such $f, g$ of class $C^{\infty}$ as follows: Define any reasonable $f=g$ in a small neighbourhood of $\pm \sqrt{2|t|}$, then extend four small $\operatorname{arcs} F_{-\frac{1}{2} f}\left(\gamma_{2(4)}\right), G_{2 g} g\left(\gamma_{1(3)}\right)$ to a curve $\bar{S}_{t}^{\prime}$ (invariant under rotation by $\pi / 2$ ) and, using also $\bar{S}_{t}^{\prime}$ symmetric to $\bar{S}_{t}$ with respect to the $x$ - or $y$-axis, find $f$ and $g$.

Is it possible to find such $f_{t}, g_{t}$ real-analytic, at least in a neighbourhood of $t=x=y=0$ ?

The whole theory from this paper holds for the resulting $H_{t}=H_{f, g}$ except for proposition 3. Can the resulting $H_{t}$ on $T^{2} \mid \mathrm{cl} U_{t}\left(U_{t}\right.$ is the domain bounded by $\left.\bar{S}_{t}\right)$ be $C^{1}$-conjugate with $\left.A\right|_{T^{2}\{\{0\}}$ ? The obstruction used in the proof of proposition 3 disappears in this case.

Remark 4. We can consider a secondary bifurcation $H_{t, s}$ of $H_{t}$. Let us start with the Hamiltonian function:

$$
h_{t, s}(x, y)=y^{2}-x^{2}\left(x^{2}+s x+2 t\right) .
$$

See the phase portrait of figure 13.

Now as in remark 3 we can look for functions $f_{t, s}, g_{t, s}, t<0$ such that $H_{f_{t}, g_{t}}$ preserves the saddles $p_{t, s}, q_{t, s}$ and a separatrix $\bar{\gamma}_{t, s}$ close to $\gamma_{t, s}$ from $p_{t, s}$ (or $q_{t, s}$ ) to itself.

Observe that we dropped the assumption from property (1) $\S 1$ that $g_{t, s}$ is an odd function, since for $s \neq 0 h_{t, s}$ is not an even function with respect to $x$.

As in remark 3 it is easy to find $f_{t, s}, g_{t, s} C^{\infty}$ for each individual $t, s$.

Is it possible to find $f_{t, s}, g_{t, s}$ real-analytic at least in a neighbourhood of $t=s=x=y=0$ ?

Are the Lyapunov exponents outside the separatrix $\gamma_{t, s}$ different from zero for $H_{t, s}, s \neq 0, t<0$ ?

The author gratefully acknowledges the financial support of the Stiflung Volkswagenwerk for a visit to the Institut des Hautes Études Scientifique during which this paper was partially written.

\section{REFERENCES}

[1] R. Burton \& R. Easton. Ergodicity of linked twist mappings. Global Theory of Dynamical Systems: Lectures Notes in Math. No. 819. Springer: Berlin, 1980, pp. 35-49.

[2] R. E. Edwards. Functional Analysis. Holt, Rinehart and Winston: New York, 1965.

[3] J. Franks. Anosov diffeomorphisms. Global Analysis. Proc. Symp. Pure Math. 14 (1970), 61-93.

[4] M. Gerber \& A. Katok. Smooth models of Thurston's pseudo-Anosov maps. Ann. Sci. de l'Ecole Normale Superieure, $4^{e}$ serie, 15 (1982), 173-204.

[5] M. Hirsch \& Ch. Pugh. Stable manifolds and hyperbolic sets. Global Analysis, Proc. Symp. Pure Math. 14 (1970), 133-163.

[6] A Katok. Bernoulli diffeomorphisms on surfaces. Ann. Math. 110 (1979), 529-547.

[7] R. Mañé. The Lyapunov exponents of generic area preserving diffeomorphisms. Preprint.

[8] Ya. B. Pesin. Lyapunov characteristic exponents and smooth ergodic theory. Uspekhi Math. Nauk. 32 No. 4 (1977), 55-112. English translation: Russ. Math. Surveys 32 No. 4 (1977), 55-114.

[9] F. Przytycki. Linked twist mappings: Ergodicity. Preprint I.H.E.S., February 1981. (To appear in part in Ann. Scientifiques de l'Ecole Norm. Sup.) 